\title{
Regulation of Mitochondrial Function by Epac2 Contributes to Acute Inflammatory Hyperalgesia
}

\author{
Diana J. Goode and Derek C. Molliver \\ Department of Biomedical Sciences, University of New England, Biddeford, Maine 04005
}

$\mathrm{G}_{\alpha \mathrm{s}}$-coupled receptors signaling through cAMP provide a key mechanism for the sensitization of nociceptive sensory neurons, and the cAMP effector Epac has been implicated in the transition from acute to chronic pain. Epac exerts its effects through Rap1 and protein kinase C (PKC). To identify targets of Epac-PKC signaling in sensory neurons of the mouse dorsal root ganglion (DRG), we profiled PKC substrate proteins phosphorylated in response to the activation of Epac with the proinflammatory prostaglandin E2 (PGE2). A prominent Epac-dependent phospho-protein band induced by PGE2 was identified by mass spectrometry as the mitochondrial enzyme pyruvate dehydrogenase (Pdha1). In dissociated DRG from both males and females, the recruitment of Pdhal to phospho-protein fractions was rapidly induced by PGE2 and prevented by selective inhibition of Epac2. Epac activation increased mitochondrial respiration, consistent with an increase in Pdhal function mediated by Epac2. Hindpaw injection of PGE2 induced heat hyperalgesia in males and females, but Pdhal phosphorylation occurred only in males. Hyperalgesia was attenuated in males but not in females by systemic inhibition of Epac2, and also by a mitochondrial membrane potential uncoupler, dinitrophenol, supporting a role for mitochondrial regulation in acute hyperalgesia. These findings identify a mechanism for the regulation of mitochondrial function by Epac2 that contributes to acute inflammatory hyperalgesia in male mice. Systemic administration of the cyclooxygenase 2 inhibitor celecoxib suppressed both PGE2-induced heat hyperalgesia and Pdhal phosphorylation in DRG of males but not females, suggesting that prostaglandin synthesis within the DRG mediates the phosphorylation of Pdhal in response to hindpaw insult.

Key words: DRG; glia; mitochondria; nociception; pain; transduction

Significance Statement

There has been extensive investigation of mitochondrial dysfunction as a causative factor in neuropathic pain disorders. In contrast, results reported here implicate enhanced mitochondrial function as a contributing factor in the development of acute inflammatory hyperalgesia. We describe a mechanism in which Epac2 activation by prostaglandin receptors leads to phosphorylation of pyruvate dehydrogenase and an increase in mitochondrial respiration in peripheral sensory neurons. Although Epac2 activation leads to Pdhal (pyruvate dehydrogenase) phosphorylation in dissociated neurons from mice of both sexes, induction of this pathway in vivo by hindpaw insult is restricted to males and appears to require intraganglionic prostaglandin synthesis. These findings support a model in which $\mathrm{G}_{\mathrm{s}}$-coupled receptor modulation of mitochondrial function promotes acute nociceptive signaling and inflammatory hyperalgesia.

\section{Introduction}

Prostaglandins are ubiquitous messengers of tissue damage that enhance nociceptive sensory neuron excitability through G-protein-coupled receptors (Ferreira and Nakamura, 1979; Lin et al.,

\footnotetext{
Received Aug. 31, 2020; revised Jan. 14, 2021; accepted Feb. 9, 2021.

Author contributions: D.J.G. and D.C.M. designed research; D.J.G. performed research; D.J.G. and D.C.M. analyzed data; D.J.G. wrote the paper.

The authors declare no competing financial interests.

Mass spectrometry was performed by the IDeA National Resource for Quantitative Proteomics at the University of Arkansas for Medical Sciences, which is supported by National Institutes of Health (NIH) Grant P20-GM-121293. This work was supported by NIH Grants R01-GM-102346, R01-NS-109936, and P20-GM103643 (to D.C.M.). We thank Ramaz Geguchadze for technical assistance.

Correspondence should be addressed to Derek C. Molliver at dmolliver@une.edu.

https://doi.org/10.1523/JNEUROSCI.2368-20.2021

Copyright $\odot 2021$ the authors
}

2006). The significance of prostaglandins for the development of pain is underscored by the extensive use of nonsteroidal antiinflammatory drugs in the United States, which provide analgesia by blocking prostaglandin synthesis (Paulose-Ram et al., 2003). Signaling by prostaglandin E2 (PGE2) in nociceptors is mediated primarily by the $\mathrm{G}_{\mathrm{s}}$-coupled receptors EP2 and EP4, which activate adenylyl cyclases to produce cAMP (Taiwo et al., 1989; Lin et al., 2006). Protein kinase A (PKA) has been broadly described as the principal effector mediating acute nociceptor sensitization by $\mathrm{G}_{\mathrm{s}}$-coupled receptors; sensitizing actions of PKA include phosphorylation of nociceptive transducers TRPV1 (transient receptor potential vanilloid type I; Hingtgen et al., 1995; Bhave et al., 2002) and TRPA1 (transient receptor potential ankyrin 1; Wang et al., 2008; Meents et al., 2017), and the tetrodotoxin-resistant $\mathrm{Na}^{+}$ channel $\mathrm{Na}_{\mathrm{v}} 1.8$ (England et al., 1996; Fitzgerald et al., 1999). 
Epac is a more recently identified effector of cAMP signaling that has physiological actions in diverse tissues (Kashima et al., 2001; Hucho et al., 2005; Fujita et al., 2017). The two isoforms of Epac, Epac1 and Epac2, are guanine nucleotide exchange factors for the Rap subfamily of small GTPases, activating Rap by exchanging GDP with GTP (Reiner and Lundquist, 2018). Epac signaling has been implicated in chronic inflammatory and neuropathic pain, but is generally thought to be dispensable for acute hyperalgesia (Wang et al., 2007; Eijkelkamp et al., 2013; Singhmar et al., 2016; Gu et al., 2016a). Epac1 has been more extensively investigated than Epac2 in chronic pain models; however, several studies report that Epac2 sensitizes nociceptors under proinflammatory conditions (Vasko et al., 2014; Shariati et al., 2016). In diverse cell types including sensory neurons, Rap1 leads to the activation of protein kinase $\mathrm{C}$ (PKC) through the production of diacylglycerol by phospholipase $\mathrm{C} \varepsilon$ (PLC $\varepsilon$; Wing et al., 2003). In particular, the $\varepsilon$ isoform of PKC (PKC $\varepsilon$ ) has been broadly implicated in nociceptor sensitization, and has been proposed as a critical link in the transition from acute to chronic pain (Reichling and Levine, 2009; $\mathrm{Gu}$ et al., 2018). Epac thus provides a mechanism through which cAMP can activate $\mathrm{PKC}$ isoforms involved in nociceptor sensitization. However, few targets of Epac-PKC signaling have been identified in sensory neurons (Eijkelkamp et al., 2013; Gu et al., 2016b; Singhmar et al., 2016).

Over the last 10 years, mitochondrial dysfunction has become a focus of active investigation for potential contributions to neuropathic pain (Flatters, 2015). A few studies have provided evidence that the normal function of mitochondria impacts nociceptor excitability (Medvedeva et al., 2008; Nita et al., 2016). To better understand contributions of Epac signaling to the genesis of pain, we characterized Epac-dependent PKC activity in response to PGE2 in mouse dorsal root ganglion (DRG) neurons. Our results suggest that Epac2 acutely enhances mitochondrial function through the PKC-dependent phosphorylation of pyruvate dehydrogenase (Pdha1) in DRG neurons, and that this mitochondrial regulation by Epac2 contributes to the hyperalgesic actions of PGE2 in male (M) mice. Identification of a role for Epac2 in the modulation of Pdhal adds a new dimension to interactions between neuronal activity and mitochondrial function.

\section{Materials and Methods}

$D R G$ isolation. DRGs were rapidly dissected and dissociated using a modified version of our previously published protocol (Malin et al., 2007). Mice were given an overdose of Avertin anesthetic and transcardial perfusion was performed with divalent ion-free HBSS on ice to clear the blood and chill the animal. DRGs were subjected to a two-step enzymatic digestion, $60 \mathrm{U}$ of papain (Worthington Biochemical) for $10 \mathrm{~min}$, followed by $2940 \mathrm{U}$ of collagenase (Worthington Biochemical) plus $15 \mathrm{U}$ of dispase II (Thermo Fisher Scientific), increased from the published protocol to $20 \mathrm{~min}$, which increases the yield of healthy neurons. After digestion, ganglia were triturated and resuspended in complete media, as follows: F12 media supplemented with 10\% fetal bovine serum and penicillin/streptomycin, $50 \mathrm{U} / \mathrm{ml}$ (Thermo Fisher Scientific). No additional trophic factors were added.

In vitro DRG stimulation. Before experiments, DRG cells were switched from complete media to HBSS for $1 \mathrm{~h}$. In some experiments, 25 $\mu \mathrm{M}$ ESI09 (an Epac1/2 inhibitor; catalog \#4773, Tocris Bioscience) or 1 mM sodium dicholoroacetate (DCA; Sigma-Aldrich) was added to the cells and incubated at $37^{\circ} \mathrm{C}$ for 20 or $10 \mathrm{~min}$, respectively, before stimulation. For the PKC $\epsilon$ inhibition experiments, PKC $\epsilon$ inhibitor peptide (500 ng $\epsilon$ V1-2, Cayman Chemical) was transported into the cells using the protocol specified by the Chariot Protein Delivery Reagent (Active Motif). Cells were stimulated with either $10 \mu \mathrm{M}$ PGE2 (catalog \#2296,
Tocris Bioscience) or $25 \mu \mathrm{M}$ 8-pCPT-2-O-Me-cAMP-AM (8pCpt; cata$\log \# 4853$, Tocris Bioscience) for $30 \mathrm{~s}, 30 \mathrm{~min}$, or $1 \mathrm{~h}$ at $37^{\circ} \mathrm{C}$. For experiments using acetyl-CoA (Sigma-Aldrich), DRG cells were incubated with $250 \mu \mathrm{m}$ acetyl-CoA with or without either $1 \mathrm{~mm}$ DCA for $1 \mathrm{~h}$ (Kerbey et al., 1976) or depolarizing HBSS containing $25 \mathrm{mM} \mathrm{K}^{+}$for the final 10 min of the acetyl-CoA application.

Protein extraction and phospho-protein enrichment. Dissociated DRG cells (in vitro assays) or acutely dissected DRG (in vivo experiments) were lysed in DiGE lysis buffer (7 M urea, $2 \mathrm{~m}$ thiourea, $4 \%$ CHAPS, $30 \mathrm{~mm}$ Tris- $\mathrm{HCl}$ ) supplemented with $1 \times$ Halt Protease and Phosphatase Inhibitor Cocktail (Thermo Fisher Scientific), and shaken at $22^{\circ} \mathrm{C}$ at $2000 \mathrm{rpm}$ for $1 \mathrm{~h}$. Lysates were quantified $(1.0 \mu \mathrm{l}$, in triplicate) with the EZQ Protein Quantification Kit (Thermo Fisher Scientific), and the same quantity of protein lysates was applied to each Fe-immobilized metal affinity chromatography (IMAC) column. $\mathrm{Fe}^{3+}$-IMAC sorbent columns were prepared with microgranular cellulose following a published protocol (Feuerstein et al., 2005). Columns packed with $800 \mu \mathrm{l}$ of $\mathrm{Fe}^{3+}$-IMAC were washed with distilled water and equilibrated with binding buffer [ $25 \mathrm{~mm}$ MES, $1 \mathrm{~m} \mathrm{NaCl}, 0.25 \%$ (w/v) CHAPS, pH 6.0]. Lysates were diluted 1:10 in binding buffer and then added to the IMAC column. Columns were washed with $2 \mathrm{ml}$ of binding buffer and eluted with $50 \mathrm{~mm}$ potassium phosphate and $50 \mathrm{~mm} \mathrm{NaCl}, \mathrm{pH}$ 7.5. Proteins were precipitated with $5 \%$ deoxycholate (Sigma-Aldrich) and trichloroacetic acid (TCA; Sigma-Aldrich) overnight at $-20^{\circ} \mathrm{C}$. Phospho-protein-enriched samples were solubilized in $1 \times$ Laemmli buffer without bromophenol blue and quantified with the EZQ Protein Quantification kit.

Western blot analysis. SDS-PAGE was performed with Bolt bis-Tris 12\% gels (Thermo Fisher Scientific) using MOPS [3-(N-morpholino) propanesulfonic acid]-SDS running buffer [500 mм MOPS, $500 \mathrm{~mm}$ Tris base, $1 \mathrm{~mm}$ EDTA, and $1 \%(\mathrm{w} / \mathrm{v})$ SDS]. Proteins were transferred to Immobilon-FL PVDF Membrane (catalog \#IPFL00010, Millipore) using Towbin buffer with SDS [250 mu Tris base, 1.92 м glycine, $1 \%$ (w/v) SDS, $10 \%$ methanol]. PVDF membranes were incubated overnight at $4^{\circ} \mathrm{C}$ with 1:500 phospho-(Ser) PKC Substrate Antibody (catalog \#2261, Cell Signaling Technology; RRID:AB_330310), 1:200 PDH-E1 $\alpha$ Antibody (D6; catalog \# sc-377092, Santa Cruz Biotechnology; RRID:AB_2716767), and 1:3000 anti-phospho-PDH-E1-A type I (Ser293; catalog \#ABS204, Millipore; RRID:AB_11205754). PVDF membrane was incubated with 1:4000 donkey anti-rabbit Cy5 (catalog \#711-175-152, Jackson ImmunoResearch) and 1:4000 donkey anti-rat Cy3 secondary antibodies (catalog \#712-165-153, Jackson ImmunoResearch) for $2 \mathrm{~h}$ at room temperature. PVDF membranes were imaged using the Typhoon 9600 laser scanner (GE Healthcare Life Sciences). Band intensities were quantified using AutoQuant imaging software.

Phospho-protein enrichment for mass spectrometry. Dissociated DRG cells were plated in six-well plates in complete media overnight. Before the experiment, DRG cells were switched from complete media to HBSS for $1 \mathrm{~h}$. ESI09 $25 \mu \mathrm{M}$ was added to half of the wells for the last $20 \mathrm{~min}$ of the incubation. Cells were stimulated with $10 \mu \mathrm{M}$ PGE2 for 30 s, then DRG cell lysates were prepared using Qiagen PhosphoProtein lysis buffer containing $0.25 \%$ CHAPS (catalog \#37101, Qiagen) and quantified using the EZQ Protein Quantification Kit. Protein concentrations were adjusted to $0.1 \mathrm{mg} / \mathrm{ml}$ and applied to the Phosphoprotein purification kit columns (catalog \#3701, Qiagen). Columns were washed with $6 \mathrm{ml}$ of PhosphoProtein lysis buffer, and phospho-protein was eluted with DiGE buffer. The concentrations of the phospho-protein eluates were determined by EZQ Protein Quantification Kit. Half of each eluate was used to run a Western blot with the phospho-(Ser) PKC substrate antibody, and the other half was used to run a "blue-silver" highresolution Coomassie stain (Candiano et al., 2004). For the blue-silver stain, the Bolt bis-Tris $12 \%$ gel was fixed with $50 \%$ ethanol and $2 \%$ phosphoric acid for $1.5 \mathrm{~h}$. The fixed gel was washed twice for $20 \mathrm{~min}$ in distilled $\mathrm{H}_{2} \mathrm{O}$, and then stained with blue-silver (10\% phosphoric acid, $10 \%$ ammonium sulfate, $5 \%$ methanol, and $0.01 \%$ Commassie G-250) overnight at room temperature. The band of interest was excised and sent to the IDeA National Resource for Quantitative Proteomics at the University of Arkansas for Medical Sciences for liquid chromatography (LC)-tandem mass spectrometry (MS/MS). 
LC-MS/MS analysis. SDS-PAGE gel slices were destained in 50\% methanol (Thermo Fisher Scientific), $100 \mathrm{~mm}$ ammonium bicarbonate (Sigma-Aldrich), followed by reduction in $10 \mathrm{~mm}$ Tris[2-carboxyethyl] phosphine (Thermo Fisher Scientific) and alkylation in 50 mм iodoacetamide (Sigma-Aldrich). Gel slices were then dehydrated in acetonitrile (Thermo Fisher Scientific), followed by the addition of $100 \mathrm{ng}$ of porcine sequencing (seq) grade modified trypsin (Promega) in $100 \mathrm{~mm}$ ammonium bicarbonate (Sigma-Aldrich) and incubation at $37^{\circ} \mathrm{C}$ for $12-16 \mathrm{~h}$. Peptide products were then acidified in $0.1 \%$ formic acid (Thermo Fisher Scientific). Tryptic peptides were separated by reverse phase Jupiter Proteo resin (Phenomenex) on a $200 \times 0.075 \mathrm{~mm}$ column using a nanoAcquity UPLC system (Waters). Peptides were eluted using a 30 min gradient from $97: 3$ to $67: 33$ buffer $A: B$ ratio (buffer $A=0.1 \%$ formic acid, $0.5 \%$ acetonitrile; buffer $\mathrm{B}=0.1 \%$ formic acid, $99.9 \%$ acetonitrile). Eluted peptides were ionized by electrospray $(2.15 \mathrm{kV})$ followed by MS/MS analysis using higher-energy collisional dissociation (HCD) on a Thermo Orbitrap Fusion Tribrid mass spectrometer in top-speed datadependent mode. MS data were acquired using the FTMS (Fouriertransform ion cyclotron resonance) analyzer in profile mode at a resolution of 240,000 over a range of 375-1500 m/z (mass-to-charge ratio). Following HCD activation, MS/MS data were acquired using the ion trap analyzer in centroid mode and normal mass range with precursor mass-dependent normalized collision energy between 28.0 and 31.0. Proteins were identified by database search using Mascot (Matrix Science) with a parent ion tolerance of $3 \mathrm{ppm}$ and a fragment ion tolerance of $0.5 \mathrm{Da}$. Scaffold 4 (Proteome Software) was used to verify MS/ MS-based peptide and protein identifications. Peptide identifications were accepted if they could be established with $<1.0 \%$ false discovery by the Scaffold Local FDR (false discovery rate) algorithm and contained at least two identified peptides. Protein probabilities were assigned by the Protein Prophet algorithm (Nesvizhskii et al., 2003). Statistics were generated using the Fisher's exact test, adjusting the FDR using the Benjamini-Hochberg procedure.

DRG preparation for Seahorse assays. DRG dissociation was performed as above. A Seahorse XF96 Cell Culture Microplate was coated with $66.7 \mu \mathrm{g} / \mathrm{ml}$ L-poly-lysine and $6.3 \mu \mathrm{g} / \mathrm{ml}$ laminin (Millipore), and plated with 5300 neurons/well. Oxygen consumption rate (OCR) was measured on a Seahorse XFe96 analyzer using the MitoStress Test kit according to manufacturer instructions. Briefly, F12 medium supplemented with $10 \%$ heat-inactivated fetal bovine serum (Thermo Fisher Scientific) and 1\% Penicillin-Streptomycin Solution (Corning) was changed to Seahorse XF Base Medium Minimal DMEM without phenol red supplemented with $1 \mathrm{~mm}$ pyruvate, $2 \mathrm{~mm}$ glutamine, and $10 \mathrm{~mm}$ glucose, $\mathrm{pH}$ 7.4. The cells were equilibrated for $30 \mathrm{~min}$ at $37^{\circ} \mathrm{C}$ in a $\mathrm{CO}_{2^{-}}$ free incubator, and then the media were replaced with the following drugs: $20 \mu \mathrm{M}$ Ce3F4 (catalog \#4793, Tocris Bioscience), $5 \mu \mathrm{M}$ HJC0350 (catalog \#4844, Tocris Bioscience), and $100 \mu \mathrm{M}$ 2,4-dinitrophenol (DNP; catalog \#34334, Sigma-Aldrich). The drugs were incubated with the DRG cells for $30 \mathrm{~min}$, and then basal respiration rates were measured. After three measurements of basal respiration, $10 \mu \mathrm{M}$ PGE2 or vehicle (Veh; DMSO) was injected with sequential injections of $2 \mu \mathrm{M}$ oligomycin, $2 \mu \mathrm{M}$ FCCP (trifluoromethoxy carbonylcyanide phenylhydrazone), and a combination of $1 \mu \mathrm{m}$ antimycin, $1 \mu \mathrm{M}$ rotenone, and $20 \mathrm{~mm}$ Hoechst dye. Respiration rates were measured three times after each treatment. After completion of the Mito Stress Test, the plate was read on the Cytation 1 (BioTek) to determine cells per well, which was used to normalize the data per manufacturer instructions (Agilent Technologies). OCR was calculated with the Seahorse XFe96 software, Wave version 2.6.

Behavioral experiments. Male and female (F) C57BL6/J mice were habituated for $1 \mathrm{~h}$ in a Plexiglas chamber. To measure withdrawal latency to noxious heat, a radiant heat source was directed at the plantar surface of the paw, and the heat source was turned off as soon as the mouse withdrew its paw (Plantar Test Analgesia Meter, Model 400 Heated Base, IITC Life Science). The elapsed time was recorded to determine the withdrawal latency. The maximum elapsed time was $30.1 \mathrm{~s}$ to prevent tissue damage. Mice received bilateral subcutaneous injections of $1 \mathrm{ng} / 20 \mu \mathrm{l}$ PGE2 in the plantar hindpaw. The following drugs were delivered by intraperitoneal injection $30 \mathrm{~min}$ before PGE2 injection: $3 \mathrm{mg} / \mathrm{kg} \mathrm{CE} 3 \mathrm{~F} 4,3 \mathrm{mg} / \mathrm{kg} \mathrm{HJC0350} \mathrm{(Tocris} \mathrm{Bioscience),} 5 \mathrm{mg} / \mathrm{kg} \mathrm{KT5720}$
Table 1. Results from one-way ANOVA statistical analyses

\begin{tabular}{llllr}
\hline & & \multicolumn{3}{l}{ Treatment } \\
\cline { 3 - 5 } Figure & Measurement (U) & dfn, dfd & $F$ & \multicolumn{1}{c}{$p$} \\
\hline $1 h$ & Pdha1 intensity (AFU) & 3,19 & 4.327 & 0.0174 \\
$2 b$ & Pdha1 intensity (AFU) & 2,10 & 45.75 & $<0.0001$ \\
$2 d$ & Pdha1 intensity (AFU) & 2,6 & 22.93 & 0.0015 \\
$3 b$ & pS293-Pdha1 intensity (AFU) & 3,16 & 13.33 & 0.0001 \\
$3 d$ & Pdha1 intensity (AFU) & 2,10 & 27.05 & $<0.0001$ \\
$4 c$ & OCR (pmol/min/normalized unit) & 3,87 & 10.25 & $<0.0001$ \\
$4 d$ & OCR (pmol/min/normalized unit) & 3,64 & 14.09 & $<0.0001$ \\
$4 f$ & Pdha1 intensity (AFU) & 2,9 & 58.97 & $<0.0001$ \\
$5 f$ & Pdha1 intensity (AFU) & 3,7 & 5.046 & 0.0359 \\
$6 e$ & Pdha1 intensity (AFU) & 2,6 & 19.35 & 0.0024 \\
$7 b$ & OCR (pmol/min/1000 cells) & 2,18 & 15.05 & 0.0001 \\
\hline
\end{tabular}

The table provides degrees of freedom, and $F$ and $p$ scores for one-way ANOVAs. dfn, df numerator; dfd, df denominator.

(Sigma-Aldrich), $30 \mathrm{mg} / \mathrm{kg}$ celecoxib (LC Laboratories), and $5 \mathrm{mg} / \mathrm{kg}$ DNP (toleration of this dose for in vivo use in mice was determined by Liu et al., 2015). The human equivalent DNP dose is $\sim 565 \mu \mathrm{g}$ (Geisler et al., 2017), which is more than two orders of magnitude lower than the dose administered to patients for obesity in the early 20th century $(\sim 300 \mathrm{mg} / \mathrm{d}$, which is toxic in some individuals; Caldeira da Silva et al., 2008). Behavioral testing was conducted $30 \mathrm{~min}$, and 1,2 , and $3 \mathrm{~h}$ after PGE2 injection.

Experimental design and statistical analyses. Experiments were performed on adult male and female C57BL6/J mice (The Jackson Laboratory). All research conformed to National Institutes of Health guidelines and procedures specified in protocols approved by the University of New England Institutional Animal Use and Care Committee. All mice received food and water ad libitum. The mass spectrometry experiment was performed on three independent pairs of samples enriched for phospho-proteins by the Qiagen PhosphoProtein Purification Kit from dissociated DRG preparations. These cultures were prepared from male mice and treated with PGE2 with or without ESI09 ( $n=3 /$ condition) for $30 \mathrm{~s}$. All other in vitro experiments used a combination of male and female mice (detailed for each experiment in Results). For all behavioral experiments, the experimenter was blinded to drug treatments.

All statistical comparisons were performed using GraphPad Prism 8 (GraphPad Software), except for the mass spectrometry analysis, which was analyzed using Scaffold 4 (Proteome Software). Data are presented as the mean \pm SEM. Significance was set at $p<0.05$ and is indicated in figures as $* p<0.05, * * p<0.01$, *** $p<0.001$, or $* * * * p<0.0001$. The exact $p$ value is reported in the Results. Before running statistical tests, the data were checked for normality (Shapiro-Wilk test), homogeneity of variances (Brown-Forsythe test), and outliers (ROUT method). Degrees of freedom, and $F$ and $p$ scores for one-way ANOVA and twoway ANOVA are presented in Table 1 and Table 2, respectively. As described in the Results, the following statistical tests were performed.

To evaluate the ESI09-dependent decrease in PGE2-induced PKC phospho-substrate staining, a paired $t$ test was used to compare the means of PGE2 and PGE2 + ESI09 (Fig. 1c).

For mass spectrometry, a Fisher's exact test with Benjamini-Hochberg multiple test correction was used to compare the total spectra (Fig. 1d) or fold change of PGE2 and PGE2 + ESI09 (Fig. 1e).

An ordinary one-way ANOVA with Tukey's multiple-comparisons test was used to evaluate the mean difference of various time frames with PGE2 (Fig. 1 $h, 1 j$ ); the effect of ESI09 (Fig. $2 b$ ) or PKC $\epsilon$ inhibitor peptide (Fig. $2 d$ ); the effect of $8 \mathrm{pCpt}$ with or without DCA on the band intensity of Pdhal (Fig. 3d), to compare the difference in mean band intensity of pS293-Pdha1 after PGE2 (Fig. 3h), after acetyl-CoA, and in the presence or absence of DCA or $25 \mathrm{~mm} \mathrm{~K}^{+}$(Fig. 3b); the mean difference of the effect of HJC0350 on the band intensity of Pdhal (Fig. 4f); the mean difference of the band intensities of Pdhal after in vivo PGE2 with or without Epac-specific inhibitors (Fig. 5f); the difference in mean band intensity of Pdhal after in vivo PGE2 with or 
Table 2. Results from two-way ANOVA repeated-measures statistical analyses

\begin{tabular}{|c|c|c|c|c|c|c|c|c|c|c|}
\hline \multirow[b]{2}{*}{ Figure } & \multirow[b]{2}{*}{ Measurement $(U)$} & \multicolumn{3}{|c|}{ Time $(0,0.5,1,2,3 \mathrm{~h})$} & \multicolumn{3}{|c|}{ Sex or treatment } & \multicolumn{3}{|c|}{ Interaction } \\
\hline & & $d f n, d f d$ & $F$ & $p$ & $d f n, d f d$ & $F$ & $p$ & $d f n, d f d$ & $F$ & $p$ \\
\hline $1 g$ & Pdha1 intensity (AFU) & 3,15 & 11.64 & 0.0003 & 1,15 & 7.901 & 0.0132 & 3,15 & 2.61 & 0.0897 \\
\hline $5 i$ & Latency (s) & 1,21 & 36.44 & $<0.0001$ & 1,21 & 2.929 & 0.1017 & 1,21 & 1.176 & 0.2905 \\
\hline $6 a$ & Latency (s) & 1,15 & 10.64 & 0.0053 & 2,15 & 3.122 & 0.0735 & 2,15 & 4.961 & 0.0222 \\
\hline $6 b$ & Latency (s) & 1,22 & 36.45 & $<0.0001$ & 1,22 & 0.3212 & 0.5766 & 1,22 & $7.74 \mathrm{E}-05$ & 0.9931 \\
\hline $7 e$ & Latency (s) & 4,32 & 12.04 & $<0.0001$ & 1,8 & 0.02345 & 0.8821 & 4,32 & 0.8958 & 0.4779 \\
\hline
\end{tabular}

The table provides degrees of freedom, and $F$ and $p$ scores for two-way ANOVAs. dfn, df numerator; dfd, df denominator.

without celecoxib (Fig. 6e,f); and the mean difference of ATP production after PGE2 with or without DNP (Fig. 7b).

An ordinary one-way ANOVA with Holm-Sidak multiple-comparisons test was used to compare the mean difference of ATP production (Fig. $4 c$ ) or maximal respiration (Fig. $4 d$ ) after PGE2 and the Epac1/2selective inhibitors.

The mean ranks of the band intensity of PKC phospho-substrate and Pdhal after in vivo injection of PGE2 or Veh (Fig. $5 b$ ) were compared using the Mann-Whitney test.

All behavior data with baseline and $1 \mathrm{~h}$ time points were analyzed by a two-way ANOVA with Sidak multiple-comparisons test. Behavioral data and in vitro PGE2 time course (Figs. $1 g, 3 g$ ) with multiple time points (baseline, $30 \mathrm{~min}$, and 1,2 , and $3 \mathrm{~h}$ ) were analyzed by a two-way ANOVA with Tukey's multiple-comparisons test.

\section{Results}

Pdha1 is phosphorylated (p) in response to Epac-dependent activation of PKC. Pronociceptive actions of PGE2 mediated by $\mathrm{G}_{\mathrm{s}^{-}}$ coupled receptors through PKA have been well documented and are thought to be mediated primarily by the EP4 receptor (Lin et al., 2006). However, potential consequences of Epac activation downstream of PGE2 signaling have not been well characterized in sensory neurons. Therefore, initial experiments were designed to detect the Epac-dependent activation of PKC in dissociated DRG preparations from male mice. Cells were stimulated with $10 \mu \mathrm{M}$ PGE2 for $30 \mathrm{~s}, 30 \mathrm{~min}$, or $1 \mathrm{~h}$, and total phospho-protein was isolated by IMAC. Samples were analyzed by Western blot using an antibody recognizing phosphorylated serine residues within the consensus amino acid sequences recognized by PKC (Nishikawa et al., 1997; Goode and Molliver, 2019). Under baseline, unstimulated conditions, several bands were consistently visualized with this antibody (referred to here as PKC p-substrate staining), indicating some constitutive PKC activity. The addition of PGE2 to cultures rapidly increased the intensity of these bands, and also induced several novel bands. Bands were more intense after $30 \mathrm{~s}$ of exposure to PGE2 and were diminished to baseline levels after $1 \mathrm{~h}$ of stimulation (Fig. 1a). To identify bands dependent on Epac signaling for induction, we applied PGE2 in the presence or absence of $25 \mu \mathrm{M}$ ESI09, which inhibits both Epac1 and Epac2, but not PKA (Almahariq et al., 2013). Epac inhibition significantly decreased the major PGE2-induced PKC psubstrate band at $\sim 41 \mathrm{kDa}$ in each of three experimental replicates [from $92.3 \pm 6.2$ to $22.1 \pm 6.5$ arbitrary fluorescence units (AFU; $p=0.0285$, paired $t$ test, $n=3$ ); Fig. $1 b, c]$. To identify the Epac-dependent phospho-protein or proteins visualized within this band, aliquots of the same six samples were run on a parallel preparative gel and stained with blue-silver stain. The region of each lane corresponding to the PKC p-substrate band was excised and processed for LC-MS/MS to compare proteins within the gel segment that were preferentially recruited to phospho-protein fractions in the presence of PGE2 compared with PGE2 plus ESI09. Figure 1d illustrates the 1136 proteins identified within this gel segment by LC-MS/MS and analyzed by Scaffold, most of which were extremely low in abundance. Only one identified protein was present in every PGE2-stimulated replicate and also significantly depleted in the presence of ESI09 (Fig. $1 e$ ), matching the Western blot analysis of these samples. Across the three independent experiments, Pdhal was 1.48-fold higher $\left[\log _{2}(1.48\right.$-fold change $\left.)=0.56\right]$ in the PGE2-stimulated condition than in cells treated with ESI09 (Fisher's exact test with Benjamini-Hochberg correction, $p=0.00000011, n=3 \mathrm{M}$ ). Amino acid sequence coverage of Pdha1 was $60-74 \%$ across all replicates.

To validate the identification of Pdhal as an Epac-dependent PKC substrate protein, we performed Pdhal Western blots to analyze total Pdhal and phospho-protein fractions obtained by IMAC enrichment from dissociated DRGs. Experiments were performed in male and female mice to determine whether PGE2-induced p-Pdhal is conserved between sexes (Fig. 1f). Similar to the results obtained with PKC p-substrate staining, a 30 s application of PGE2 significantly increased Pdha1 band intensity in phospho-protein fractions in male (from $27.89 \pm 1.5$ to $63.16 \pm 5.0$ AFU, $p=0.0314$, two-way ANOVA, $n=3$ ) and female mice (from $28.98 \pm 1.5$ to $66.29 \pm 12.24$ AFU, $p=0.0272$, two-way ANOVA, $n=3$ ). Interestingly, PGE2-induced p-Pdhal was somewhat more prolonged in female mice, and Pdhal band intensity peaked after 30 min of PGE2 exposure (87.58 \pm 11.0 AFU, $p=0.0006$, two-way ANOVA, $n=3$ ). The band intensity of p-Pdhal decreased from peak after $1 \mathrm{~h}$ in both males (from $63.16 \pm 5.0$ to $28.1 \pm 1.9 \mathrm{AFU}, p=0.0227$, two-way ANOVA, $n=3$ ), and females (from $87.58 \pm 11.0$ to $48.76 \pm 5.94 \mathrm{AFU}$, $p=0.0207$, two-way ANOVA, $n=3$ ). The only time point at which the band intensity of Pdhal was significantly different between male and female mice was after 30 min PGE2 exposure (female, 87.58 $\pm 11.0 \mathrm{AFU}$; male $47.53 \pm 11.63 \mathrm{AFU} ; p=0.0110$, two-way ANOVA, $n=3$ /sex; Fig. $1 g$ ). With the PGE2-induced pPdhal effect similar in males and females, we combined the data [Fig. $1 h ; n=6$ (3 M, $3 \mathrm{~F}$, combined)]. For subsequent in vitro experiments, we used a 30 s PGE2 stimulation and combined data from male and female mice. In contrast to the increase in $\mathrm{p}$ Pdha1 in response to PGE2, there was no change in total Pdha1 band intensity (normalized to tubulin) from total pre-enriched (unfractionated) samples during the time course of the experiment [Fig. 1i,j; $n=5$ (2 M, 3 F, combined)]. Finally, like the PKC p-substrate staining, Epac signaling was required for the recruitment of Pdhal to phospho-protein fractions, as follows: PGE2 significantly increased Pdhal band intensity (to 82.2 $\pm 7.0 \mathrm{AFU}$ ) from baseline ( $3.1 \pm 1.6 \mathrm{AFU})$, and this increase was inhibited by ESI09 [14.0 \pm 6.3 AFU, Fig. $2 a, b ; p<0.0001$, one-way ANOVA, $n=5$ (3 M, 2 F, combined)]. 
a.

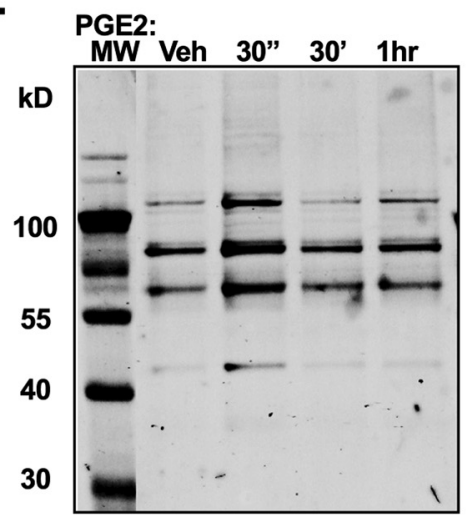

d.

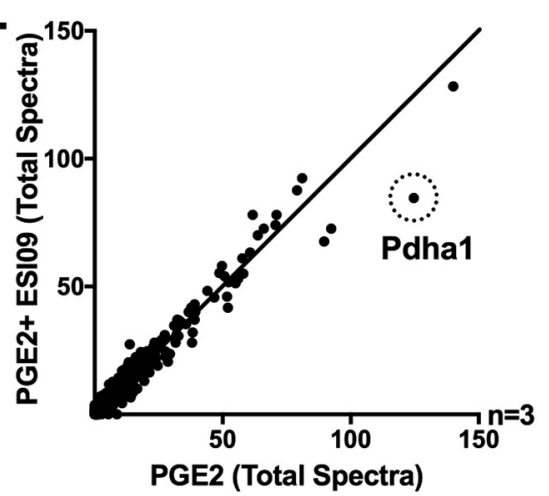

f.

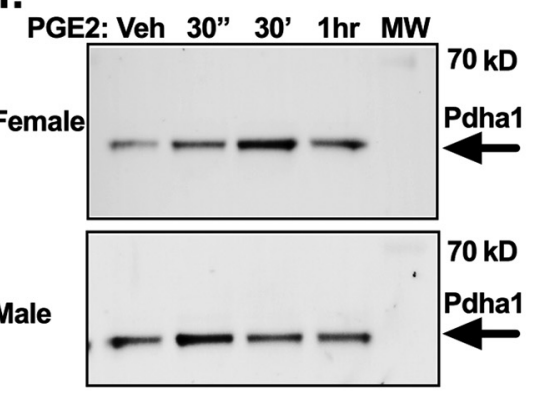

i.

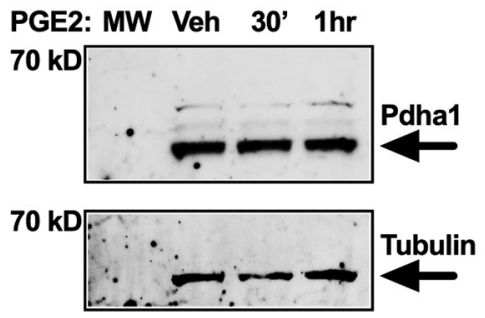

b.

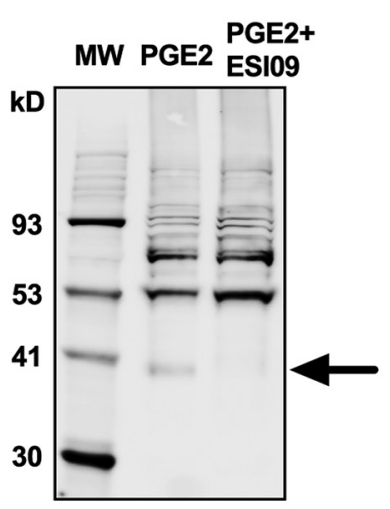

e.

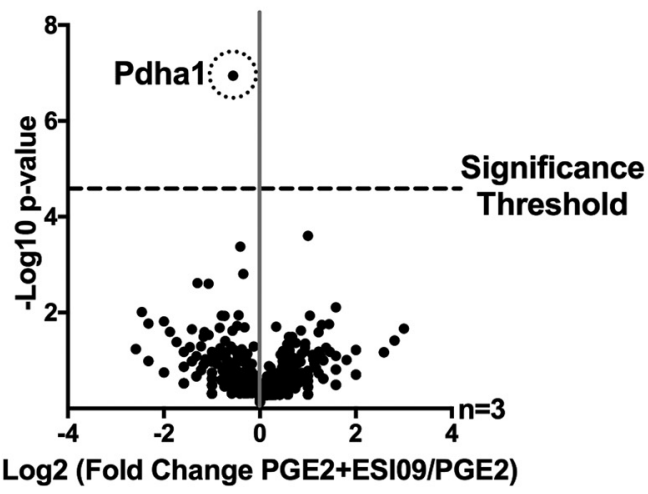

c.

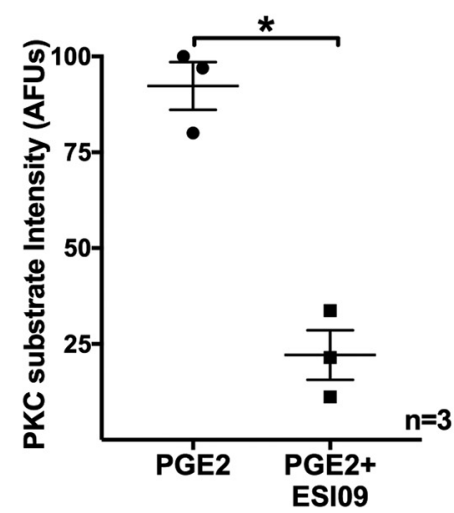

g.

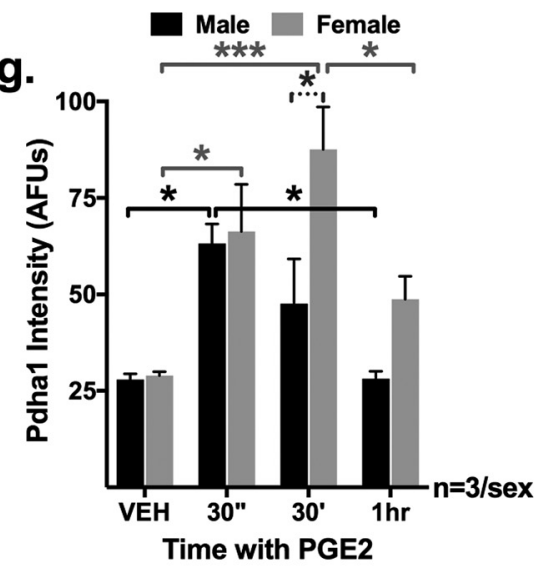

j.

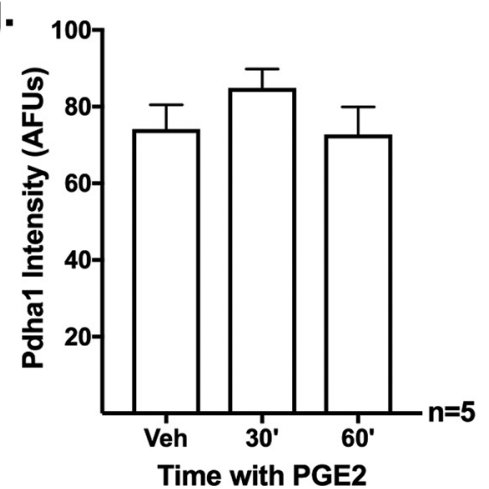

h.

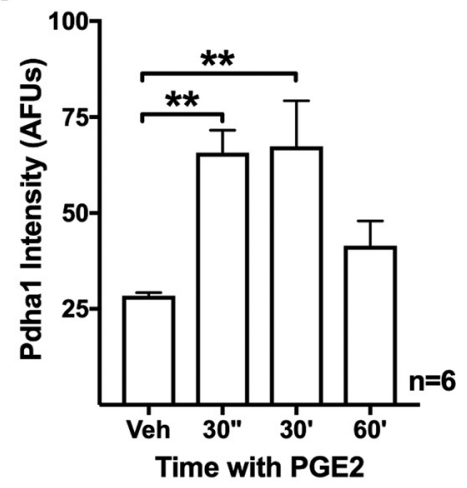

Figure 1. Pdha1 is phosphorylated in response to Epac-dependent activation of PKC. $\boldsymbol{a}, \boldsymbol{b}$, PKC p-substrate profile produced by Western blot of samples enriched for phospho-protein by IMAC from dissociated DRG neurons stimulated with $10 \mu \mathrm{M}$ PGE2 (30 s, $30 \mathrm{~min}$, and $1 \mathrm{~h}$; $\boldsymbol{a}$ ) or PGE2 plus $25 \mu \mathrm{m}$ Epac inhibitor ESI09 (b) and probed with the PKC p-substrate antibody. A major PGE2-induced band of $\sim 41 \mathrm{kDa}$ was suppressed by ESI09 [black arrow; molecular weight markers (MW)]. c, Quantification of the $41 \mathrm{kDa}$ band intensity over three replicates. Statistical significance was determined by Student's paired $t$ test $(* p<0.05)$. $\boldsymbol{d}, \boldsymbol{e}$, Identification of Pdha1 by LC-MS. Separate aliquots of the same phospho-protein samples analyzed in $\mathbf{c}$ were separated by PAGE and visualized with blue-silver stain. The region of the gel corresponding to the ESI09-sensitive band was excised from each lane and analyzed by LC-MS/MS in triplicate. $\boldsymbol{d}$, Each dot 
a.

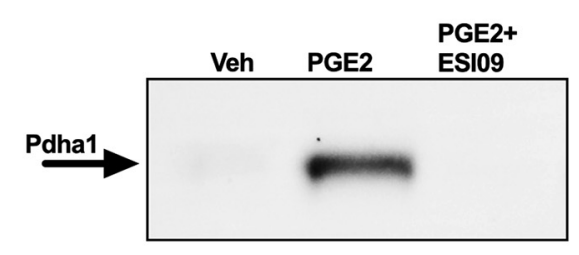

C.

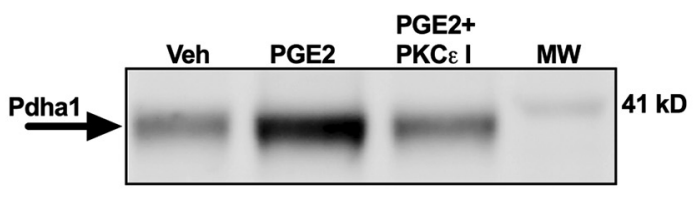

b.

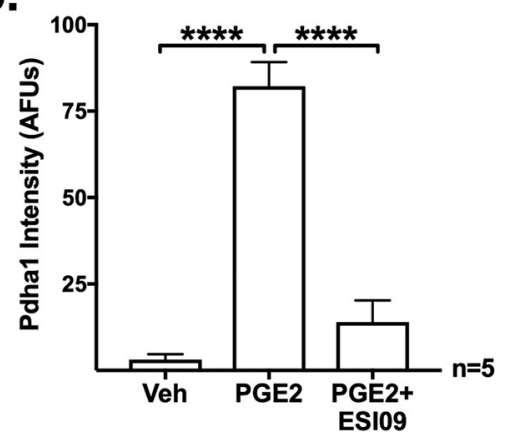

d.

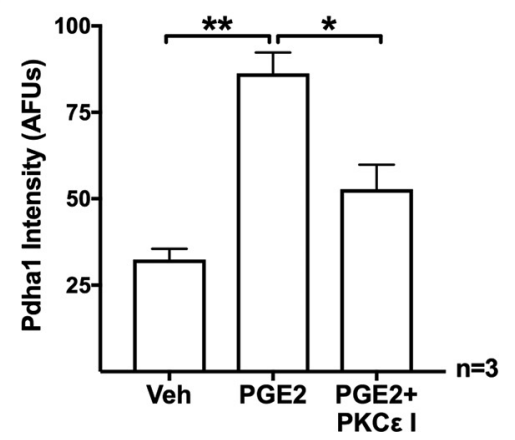

Figure 2. Phosphorylation of Pdha1 in response to PGE2 requires Epac and PKC $\varepsilon$ activity. $\boldsymbol{a}$, Western blot of phospho-enriched samples from dissociated DRG neurons stimulated with 10 $\mu \mathrm{m}$ PGE2 with or without $25 \mu \mathrm{m}$ ESI09, probed with an antibody to Pdha1. $\boldsymbol{b}$, The bar graph quantifies Pdha1 signal intensity in phospho-enriched fractions after PGE2 stimulation in the presence or absence of ESI09. Statistical significance was determined by ordinary one-way ANOVA with Tukey's multiple-comparisons test. $* * * * p<0.0001, n=5$ ( $3 \mathrm{M}, 2 \mathrm{~F}$ ). c, Pdha1 Western blot of phospho-enriched samples from dissociated DRG neurons stimulated with $10 \mu \mathrm{m}$ PGE2 (30 s) with or without $250 \mu \mathrm{g}$ of PKC $\varepsilon$ inhibitor peptide $\varepsilon$ V1-2 (PKC $\varepsilon$ I). $\boldsymbol{d}$, Quantification of the intensity of PGE2-induced Pdha1 bands in phospho-enriched samples after PKC $\varepsilon$ inhibition. Statistical significance was determined by ordinary one-way ANOVA with Tukey's multiple-comparisons test $[* p<0.05, * * p<0.01, n=3(2 \mathrm{M}, 1 \mathrm{~F})]$.

Identification of Pdha1 by PKC p-substrate Western blot indicates that Pdhal phosphorylation is likely mediated by PKC; however, it is possible that the antibody recognized a $\mathrm{p}$-serine mediated by a different kinase. A number of PKC isoforms are expressed in mouse DRG neurons; however, PKC $\varepsilon$ has been reported to be activated by Epac, and to be required for prolonged hyperalgesia (Aley et al., 2000; Parada et al., 2003). Inhibition of PKC $\varepsilon$ with $250 \mu \mathrm{g}$ of $\varepsilon \mathrm{V} 1-2$ (PKC $\varepsilon$ inhibitor peptide) decreased PGE2-induced p-Pdhal by $62.2 \%$ [from $86.3 \pm$ 6.1 to $52.8 \pm 7.1 \mathrm{AFU}, p=0.0139$, one-way ANOVA, $n=3(2 \mathrm{M}$, $1 \mathrm{~F}$, combined); Fig. $2 c, d$ ], implicating $\mathrm{PKC} \varepsilon$ as the major kinase contributing to PGE2-induced p-Pdha1.

represents a protein ID quantified on the basis of total spectrum count. The diagonal line represents theoretical equal abundance between the two conditions. Pdha1 showed the largest decrease in abundance from phospho-protein-enriched samples in response to ESIOO of any protein identified. $\boldsymbol{e}$, Volcano plot comparing the mean fold change to significance, determined by the Fisher's exact test with Benjamini-Hochberg correction. The horizontal dotted line indicates the significance threshold. Pdha1 was the only ID that was significantly diminished in the presence of ESI09 ( $p<0.00002$ for Pdha1). $\boldsymbol{f}$, An antibody to Pdha1 was used to probe Western blots of phospho-enriched samples from dissociated DRG after $10 \mu \mathrm{m}$ PGE2 stimulation [baseline (BL), $30 \mathrm{~s}, 30 \mathrm{~min}$, and $1 \mathrm{~h}$ of stimulation]. $\boldsymbol{g}$, Quantification of the intensity of Pdha1 bands in $\boldsymbol{f}$ from male and female mice $(n=3 / \mathrm{sex})$. $\boldsymbol{h}$, Male and female data from $\boldsymbol{g}$ combined $[n=6(3 \mathrm{M}, 3 \mathrm{~F})]$. Statistical significance was determined by two-way ANOVA with Tukey's multiple-comparisons test $(* p<0.05, * * p<0.01$, $* * * p<0.001)$. $\boldsymbol{i}$, Western blot of total Pdha1 and tubulin from unfractionated DRG samples after 30 and 60 min stimulation with $10 \mu \mathrm{M}$ PGE2. $j$, Quantification of the intensity of Pdha1 bands normalized to tubulin $[n=5(2 \mathrm{M}, 3 \mathrm{~F})]$.
Pdha1, which catalyzes the irreversible conversion of pyruvate to acetyl-CoA, is tightly regulated by dedicated pyruvate dehydrogenase kinases (PDKs) and opposing pyruvate dehydrogenase phosphatases (PDPs; Linn et al., 1969; Cook et al., 1984; Paxton et al., 1986). These dual control mechanisms allow the cell to balance the opposing needs of ATP production and nutrient conservation. PDKs phosphorylate Pdhal at three sites (S293, S300, and S232) to inhibit enzymatic activity. Phosphorylation of S293 is particularly effective at inactivating the enzyme, and therefore to detect the phosphorylation of Pdhal by PDKs, we probed Western blots with an antibody selective for p-S293-Pdha1. PDK activity was stimulated by incubation with $250 \mu \mathrm{M}$ acetyl-CoA, the product of Pdha1, based on the findings of a previous study (Kerbey et al., 1976). The PDK inhibitor DCA was used at $1 \mathrm{~mm}$ to demonstrate inhibition of S293-Pdhal phosphorylation (Kerbey et al., 1976; Bonnet et al., 2007). We also tested the ability of $\mathrm{Ca}^{2+}$ influx (induced by depolarization with buffer containing $25 \mathrm{mM} \mathrm{K}^{+}$for $10 \mathrm{~min}$ ) to reverse Pdhal phosphorylation through the activation of PDP (Huang et al., 1998; Fig. 3a,b). Compared with normal complete media, media containing acetyl-CoA induced a twofold increase in the intensity of the pS293-Pdhal band after $1 \mathrm{~h}$ of exposure [from $42.4 \pm 9.0$ to 94.6 $\pm 2.7 \mathrm{AFU}$; normalized to total Pdha1, $p=0.0001$, one-way ANOVA, $n=5$ ( $2 \mathrm{M}, 3 \mathrm{~F}$, combined)]. The effect of acetyl-CoA was almost entirely eliminated when DCA was included with the acetyl-CoA [50.8 $\pm 16.9, p=0.0008$, one-way ANOVA, $n=5$ (2 $\mathrm{M}, 3 \mathrm{~F}$, combined)] or by a $10 \mathrm{~min}$ exposure to $25 \mathrm{mM} \mathrm{K}^{+}$for the last $10 \mathrm{~min}$ of the acetyl-CoA application [58.1 $\pm 3.6, p=0.0042$, one-way ANOVA, $n=5$ ( $2 \mathrm{M}, 3 \mathrm{~F}$, combined)]. To rule out the possibility that the increase in p-Pdhal induced by PGE2 could 
a.

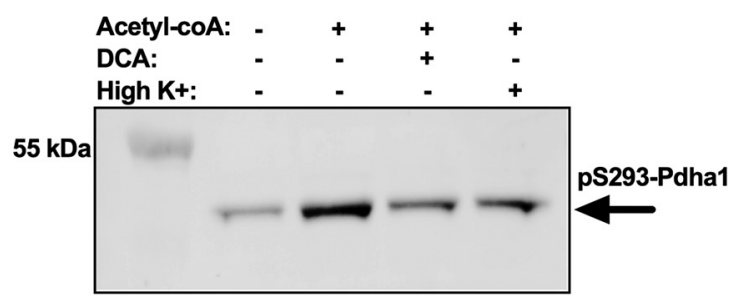

c.

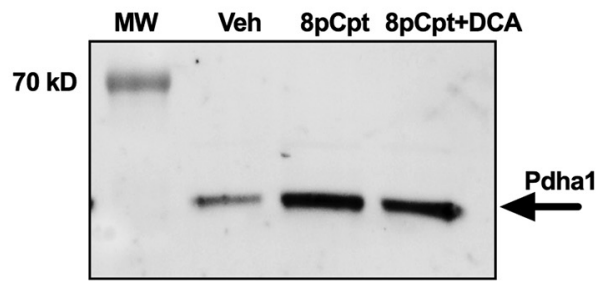

e.

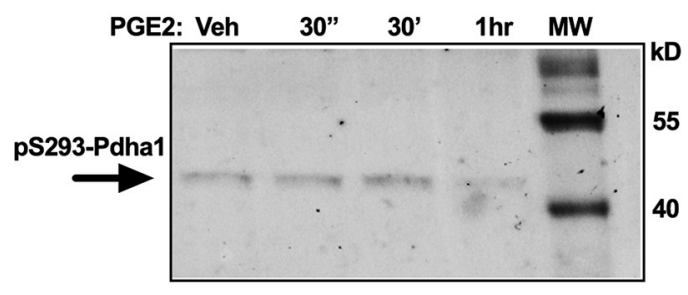

g.

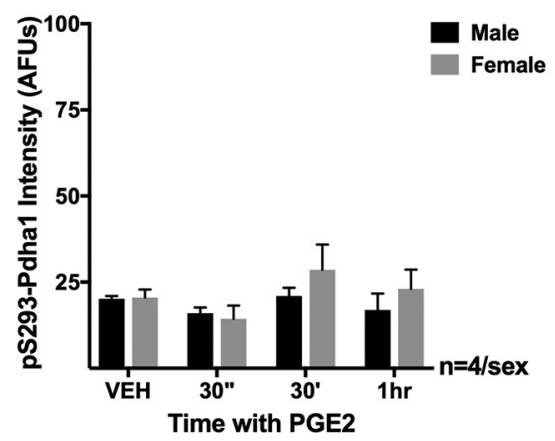

b.

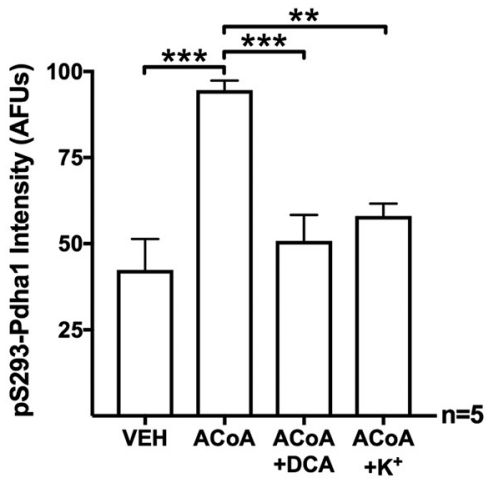

d.

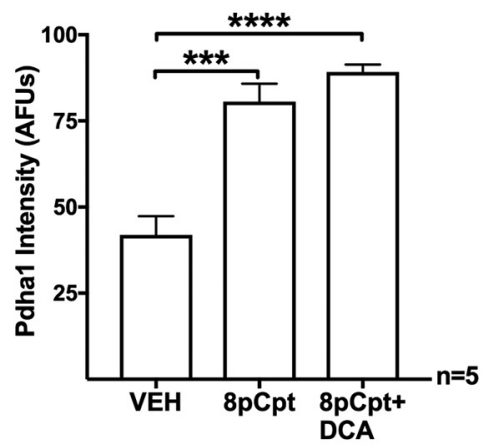

f.

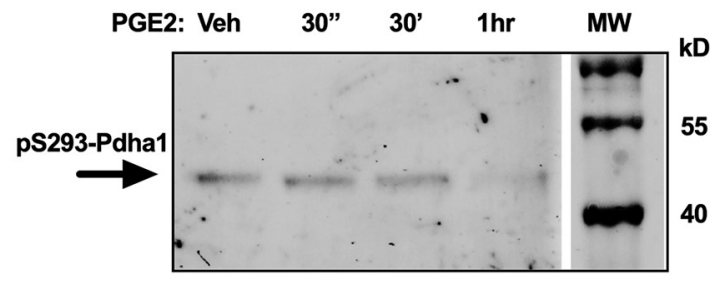

h.

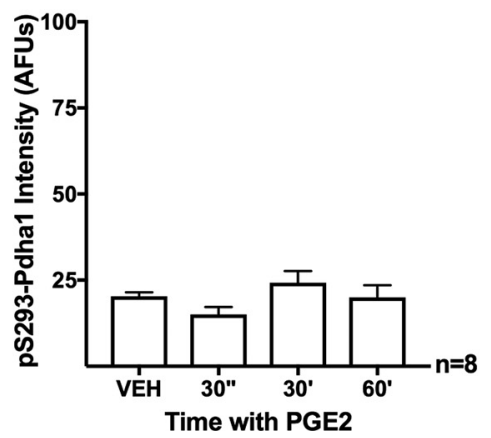

Figure 3. Epac-dependent phosphorylation of Pdha1 is distinct from phosphorylation by PDK. $\boldsymbol{a}$, Western blot of dissociated DRG neurons incubated with $250 \mu \mathrm{m}$ acetyl-coA in the presence or absence of $1 \mathrm{~mm} \mathrm{DCA}(1 \mathrm{~h})$ or $25 \mathrm{~mm} \mathrm{~K}^{+}$(10 min). $\boldsymbol{b}$, Quantification of the pS293-Pdha1 band intensities (normalized to total Pdha1) from $\boldsymbol{a}$. Acetyl-CoA stimulated an increase in pS293Pdha1 intensity that was largely reversed in the presence of either DCA or $25 \mathrm{~mm} \mathrm{~K}^{+}$. Statistical significance was determined by one-way ANOVA with Tukey's multiple-comparisons test $[* * * p<0.001, * * p<0.01, n=5(2 \mathrm{M}, 3 \mathrm{~F})]$. c, Western blot of phospho-enriched fractions from dissociated DRG neurons stimulated for $30 \mathrm{~s}$ with Epac activator 8pCpt with or without PDK inhibitor DCA (1 mm; $10 \mathrm{~min})$. d, Quantification of Pdha1 band intensity. DCA did not diminish Pdha1 recruitment to phospho-enriched fractions. Statistical significance was determined by one-way ANOVA with Tukey's multiple-comparisons test [***p $<0.001, * * * * p<0.0001, n=5(2 \mathrm{M}, 3 \mathrm{~F})]$. $\boldsymbol{e}, \boldsymbol{f}$, pS293-Pdha1 Western blot of phospho-enriched samples from dissociated DRG neurons from females $(\boldsymbol{e})$ and males $(\boldsymbol{f})$ after stimulation with $10 \mu \mathrm{m}$ PGE2 injection [baseline (BL), $30 \mathrm{~s}, 30 \mathrm{~min}$, and $1 \mathrm{~h}$ ]. $\boldsymbol{g}$, Quantification of the signal intensity of pS293-Pdha1 bands in $\boldsymbol{e}$ and $\boldsymbol{f}$. $\boldsymbol{h}$, Quantification of the signal intensity of combined male and female data in $\boldsymbol{g}$. PGE2 did not alter the phosphorylation of the PDK substrate residue S293-Pdha1.

be mediated by PDK activation, we used an activator of both Epac1 and Epac2, 8pCpt $(25 \mu \mathrm{M})$ in the presence or absence of DCA. 8pCpt doubled baseline p-Pdhal intensity from $41.8 \pm 5.5$ to $80.6 \pm 11.7 \mathrm{AFU}[p=0.0004$, one-way ANOVA, $n=5(2 \mathrm{M}, 3$ F, combined); Fig. $3 c, d]$. However, DCA (75.7 $\pm 6.6 \mathrm{AFU})$ had no effect on the increase in p-Pdhal band intensity induced by $8 \mathrm{pCpt}[p=\mathrm{ns}$, one-way ANOVA, $n=5(2 \mathrm{M}, 3 \mathrm{~F}$, combined $)]$. Furthermore, despite the increase in total Pdhal phosphorylation induced by PGE2, PGE2 did not alter p-S293-Pdha1 intensity at any time point tested in males (Fig. $3 e, g$ ) or females (Fig. 
a.

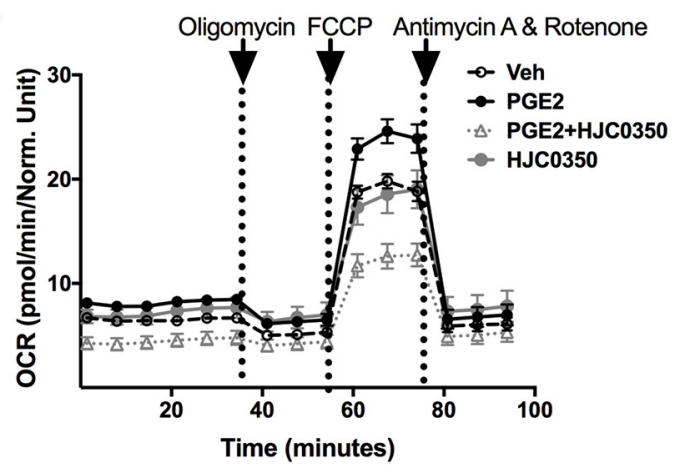

c.

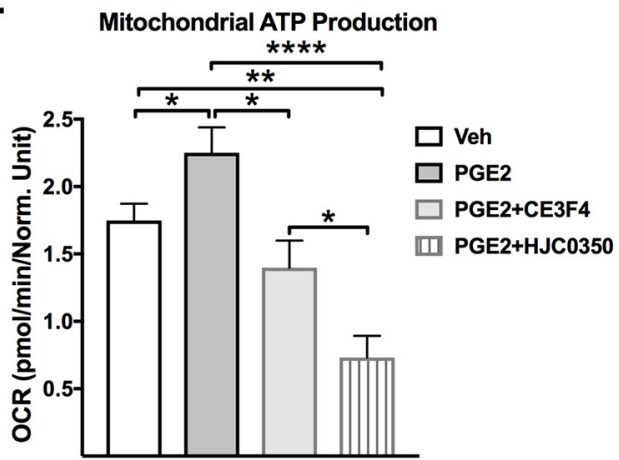

e.

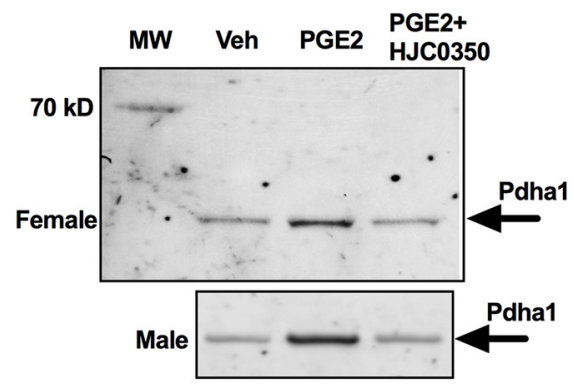

g.

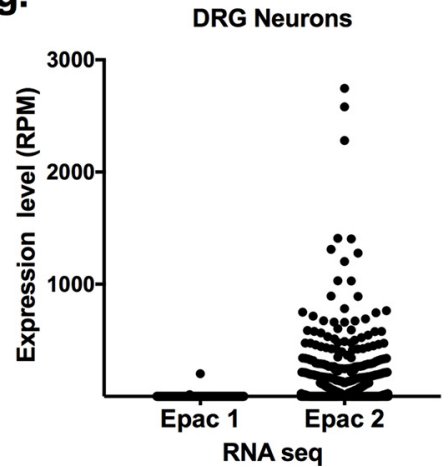

b.

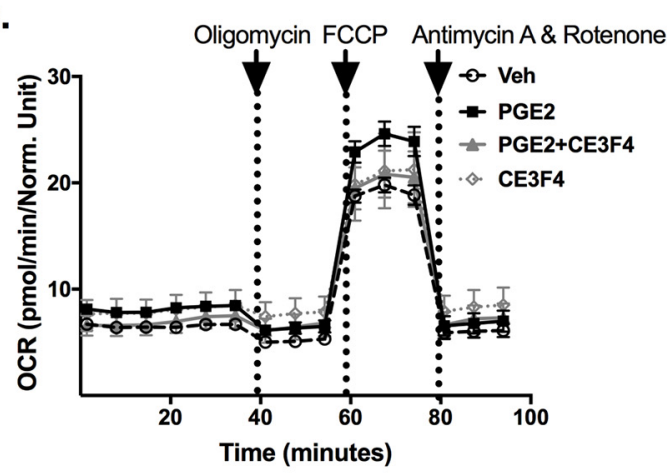

d.

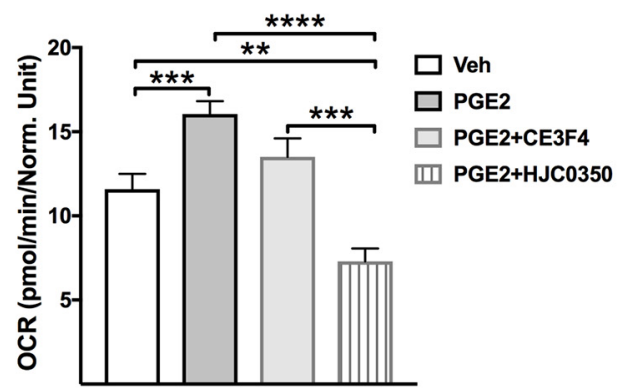

f.

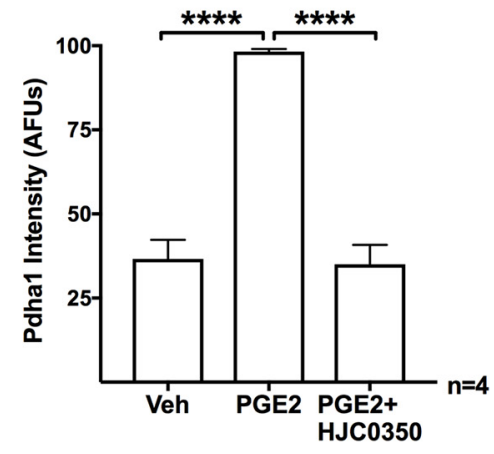

h.

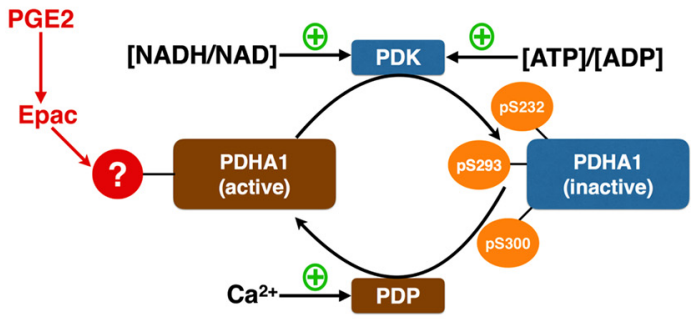

Figure 4. PGE2 increases mitochondrial respiration through Epac2. Measurement of mitochondrial respiration, determined by the OCR using the Seahorse Mito Stress Test in dissociated DRGs. $\boldsymbol{a}, \boldsymbol{b}$, shows the OCR of cells stimulated with $10 \mu \mathrm{M}$ PGE2 with or without $5 \mu \mathrm{M} \mathrm{HJC0350} \mathrm{(Epac2-selective} \mathrm{inhibitor;} \boldsymbol{a}$ ) or $20 \mu \mathrm{m}$ Ce3F4 (Epac1-selective inhibitor; $\boldsymbol{b}$ ) in the presence of Mito Stress Test drugs (oligomycin inhibits ATP synthase, FCCP collapses the $\mathrm{H}^{+}$gradient, rotenone + antimycin inhibits Complex I/III). $c$, Changes in the OCR attributable to ATP production in

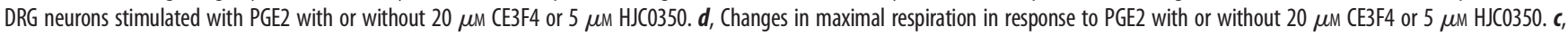
$\boldsymbol{d}$, Statistical significance was determined by ordinary one-way ANOVA with Holm-Sidak multiple-comparisons test. $* p<0.05 ; * * p<0.01 ; * * * p<0.001 ; * * * * p<0.0001$; replicates $($ Veh $=37$, PGE2 $=31$, PGE2 + HJC0350 $=12$, PGE2 + CE3F4 = 12) across multiple plates were combined and normalized (Norm.) to Veh control. e, Western blot of phospho-enriched samples from dissociated DRG neurons from female (top blot) and male (bottom blot) mice stimulated with $10 \mu \mathrm{m}$ PGE2 with or without $5 \mu \mathrm{m}$ HJC0350, probed with an antibody to Pdha1. $f$, The bar graph quantifies Pdha1 signal intensity in phospho-enriched fractions after PGE2 stimulation in the presence or absence of HJC0350 ( $n=4 ; 2 \mathrm{~F}, 2 \mathrm{M}$, combined). Statistical significance was determined by ordinary one-way ANOVA with Tukey's multiple-comparisons test. $* * * * p<0.0001$. , We analyzed Epac1 and Epac2 mRNA levels [reads per million (RPM)] in a previously 
$3 f, g$ ), or when results from males and females were combined (Fig. 3h). These results indicate that the phosphorylation of Pdhal in response to the activation of Epac is not mediated by PDKs and is therefore a distinct mechanism from the canonical regulation of Pdha1 function.

To determine the functional consequences of Epac2-dependent phosphorylation of Pdha1, we performed metabolic flux analysis using the Seahorse Analyzer (Agilent Technologies). This assay measures mitochondrial function by quantifying the OCR of dissociated DRG cells in the presence of drugs that target selected components of mitochondrial function, as follows: oligomycin inhibits ATP synthase, allowing measurement of the amount of oxygen consumption dedicated to ATP production; FCCP dissipates the mitochondrial proton gradient, forcing mitochondria to undergo maximal respiration; rotenone inhibits complex I and antimycin inhibits complex III; together they eliminate mitochondrial respiration, allowing the detection of any nonmitochondrial oxygen consumption. Selective inhibitors for Epac1 and Epac2 were included to determine which Epac isoform mediates the effects of PGE2.

Application of $10 \mu \mathrm{M}$ PGE2 increased the OCR in dissociated DRGs [Fig. $4 a ; n=4$ (2 M, $2 \mathrm{~F}$, combined)]. This increase in OCR was prevented by the Epac2 inhibitor HJC0350 (5 $\mu \mathrm{M}$; Fig. $4 a$ ), but not the Epac1 inhibitor CE3F4 (20 $\mu \mathrm{M}$; Fig. $4 b)$. HJC0350 did not alter OCR in the absence of PGE2. The PGE2induced increase in OCR included a significant increase in both the OCR attributable to ATP production (ATP synthase activity; Fig. $4 c$ ) and the maximal respiration rate (Fig. $4 d$ ). ATP synthase activity in unstimulated DRG cultures produced $1.75 \pm 0.12$ OCR, and this increased by $>28 \%$ with PGE2, to $2.25 \pm 0.19$ OCR $[p=0.0484$, one-way ANOVA; technical replicates $(\mathrm{Veh}=37, \mathrm{PGE} 2=31$, PGE2 + HJC0350 = 12, PGE2+CE3F4 = 12) across multiple plates were combined and normalized to Veh control]. HJC0350 completely reversed the PGE2-induced ATP production, from $2.25 \pm$ 0.19 to $0.73 \pm 0.16$ OCR ( $p<0.0001$, one-way ANOVA; technical replicates: PGE2 $=31, \mathrm{PGE} 2+\mathrm{HJC} 0350=12$ ). In contrast, $\mathrm{CE} 3 \mathrm{~F} 4$ did not alter ATP production compared with unstimulated DRG cultures. (Fig. $4 c, d$ ). The maximal respiratory capacity of DRG cells, revealed by the application of FCCP, was increased $>27 \%$ by PGE2, from $11.58 \pm 1.2$ OCR in unstimulated cells to $16.05 \pm 1.1$ OCR $(p=0.0010$, one-way ANOVA; technical replicates: Veh $=37$, PGE2 =31). The increase in maximal respiration in response to PGE2 is consistent with an increase in Pdhal function because the rate of respiration is limited by the availability of acetyl-CoA produced by Pdha1. There was no significant change in maximal respiration between DRG cells treated with PGE2 and cells treated with PGE2 and CE3F4, confirming that Epac1 does not contribute to the effect of PGE2 on mitochondrial respiration under these conditions.

HJC0350 also prevented the PGE2-induced recruitment of Pdhal to IMAC fractions, visualized by Pdhal Western blot in DRG cells from female and male mice $[p<0.0001$, one-way ANOVA, $n=4$ (2 M, 2F, combined); Fig. 4e,f], demonstrating that the increase in OCR mediated by Epac2 is associated with

$\leftarrow$

published single-cell RNA-seq dataset (Usoskin et al., 2015). Data were collected from 622 isolated DRG neurons from L4-6 DRG of naive 6- to 8-week-old male and female C57BL/6 mice. Each dot represents the normalized expression level in RPM for an individual neuron. $\boldsymbol{h}$, The cartoon illustrates the canonical regulation of Pdha1 by PDKs and PDPs, as well as our results suggesting that Epac2-PKC $\varepsilon$ signaling provides a novel mechanism for the regulation of Pdha1 function distinct from the canonical pathway. Based on information from the study by Sugden and Holness (2011). MW, Molecular weight. the phosphorylation of Pdha1. The selectivity of this response for Epac2 over Epac1 may be because of the limited expression of Epac1 in naive neurons; our analysis of published single-cell mRNA-seq data (Usoskin et al., 2015) revealed that Epac1 is expressed at very low levels in naive, acutely dissociated DRG neurons, whereas Epac2 is broadly expressed (Fig. 4g). Together, these results suggest that Epac2-dependent activation of PKC leads to the phosphorylation of a noncanonical residue or residues on Pdhal that positively regulate mitochondrial respiration (Fig. 4h).

The physiological significance of Epac2 signaling was next examined in vivo, by injecting $1 \mathrm{ng} / 20 \mu \mathrm{l}$ PGE2 into the plantar hindpaw of male mice and measuring paw withdrawal latencies to noxious heat. PGE2 injection significantly reduced latencies $60 \mathrm{~min}$ after injection, from $20.0 \pm 1.39$ to $7.621 \pm 1.10 \mathrm{~s}$ $(p=0.0002$, two-way ANOVA, $n=8$; Fig. $5 a)$. Systemic administration of the Epac1 inhibitor CE3F4 (3 mg/kg) $30 \mathrm{~min}$ before hindpaw PGE2 injection had no significant effect on PGE2-induced hyperalgesia ( $p=\mathrm{ns}$, two-way ANOVA, $n=8$ ). However, systemic injection of $3 \mathrm{mg} / \mathrm{kg} \mathrm{HJC0350} \mathrm{prevented} \mathrm{hyperalgesia} \mathrm{(PGE2,}$ $7.62 \pm 1.23 \mathrm{~s}$; vs PGE2 + HJC0350, $16.76 \pm 2.88 \mathrm{~s} ; p=0.0090$, twoway ANOVA, $n=8$ ). Inhibition of PKA with $5 \mathrm{mg} / \mathrm{kg}$ KT 5720 (Kase et al., 1987) also prevented PGE2-induced hyperalgesia.

Following behavioral analysis, L3-5 DRGs were rapidly dissected to determine the phosphorylation state of Pdha1. Hindpaw PGE2 injection increased p-Pdhal in DRGs by $52.73 \%$ by Pdhal Western blot $(p=0.0006$, Mann-Whitney test, $n=8)$ and by $62.02 \%$ by PKC $p$-substrate Western blot $(p=0.0040$, MannWhitney test, $n=5 \mathrm{M}$; Fig. $5 b$ ). These results likely underestimate the magnitude of the response because of signal dilution, because only a subset of neurons in L3-5 DRGs innervate the hindpaw. To confirm that the increase in p-Pdhal in vivo was not because of an increase in PDK activity or in total Pdhal levels, we quantified pS293-Pdhal and Pdhal in total protein (unfractionated) samples from L3-5 DRG, $1 \mathrm{~h}$ after hindpaw injection of PGE2 or vehicle. PGE2 did not alter p-S293-Pdha1 or total Pdha1 (normalized to tubulin; Fig. $5 c, d$ ). Systemic inhibition of Epac2, but not Epac1, significantly inhibited p-Pdhal $(57.44 \% \pm 13.49)$ in phospho-protein fractions $1 \mathrm{~h}$ after hindpaw injection of PGE2 $(p=0.0445$, one-way ANOVA, $n=3$ M; Fig. 5e,f). Analysis of p-S293-Pdha1 in these same samples revealed that neither PGE2 injection nor Epac inhibitors altered pS293-Pdhal band intensity ( $p=n s$, one-way ANOVA, $n=3 \mathrm{M}$; Fig. $5 g, h)$. Thus, in males, PGE2 injection evokes heat hyperalgesia that coincides with Epac2-dependent, PDK-independent phosphorylation of Pdha1, consistent with the effect of PGE2 in vitro. However, in contrast to the effects of HJC0350 in male mice, the administration of HJC0350 in females did not reduce PGE2-induced heat hyperalgesia ( $p=$ ns, two-way ANOVA, $n=11$ F; Fig. 5i), and the phosphorylation state of Pdhal was not altered by PGE2 with or without HJC0350 ( $p=n s$, two-way ANOVA, $n=3$ F; Fig. $5 j, k$ ).

These results raise the question, how does hindpaw injection of PGE2 cause the rapid induction of p-Pdha1 in the DRG, given insufficient time for the retrograde transport of cell-signaling molecules from the axon terminals? We hypothesized that the induction of p-Pdhal must be mediated by local signaling events at the cell body induced by action potential firing from the terminals. It has been reported that satellite glial cells (SGCs) produce prostaglandins in response to nociceptor activation (Souza et al., 2013), and multiple roles for SGCs in nociceptor sensitization have been described (Hanani, 2012; Ferrari et al., 2014; Christie et al., 2015). These reports suggest that nociceptor firing could rapidly lead to prostaglandin synthesis within the ganglia 
a.

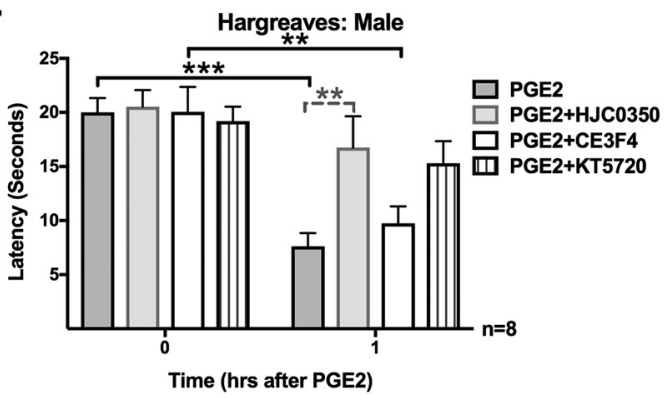

c.

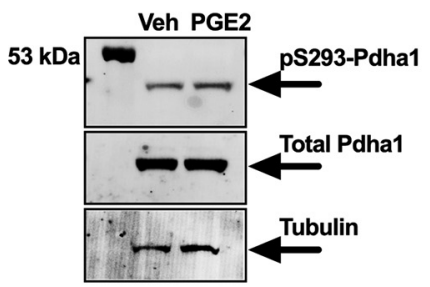

e.

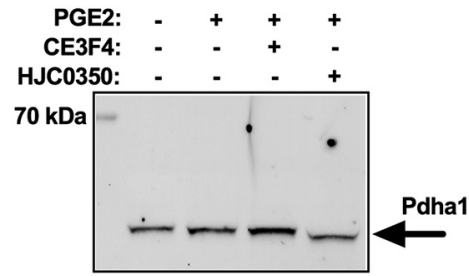

g.

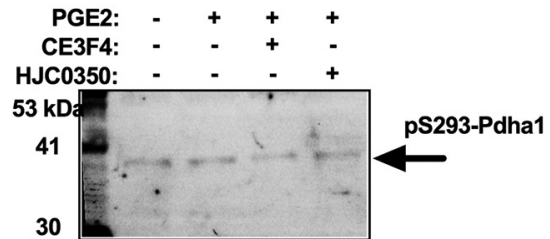

i.

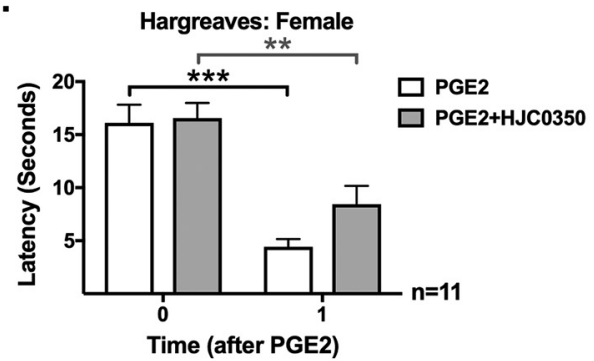

k.

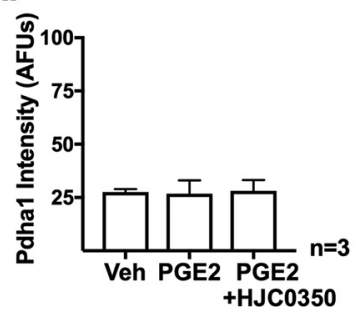

b.

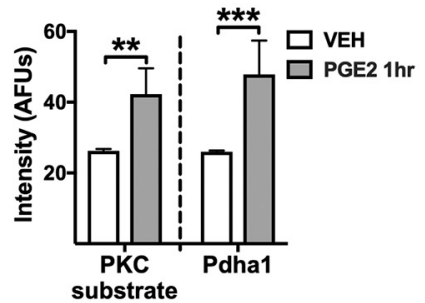

d.

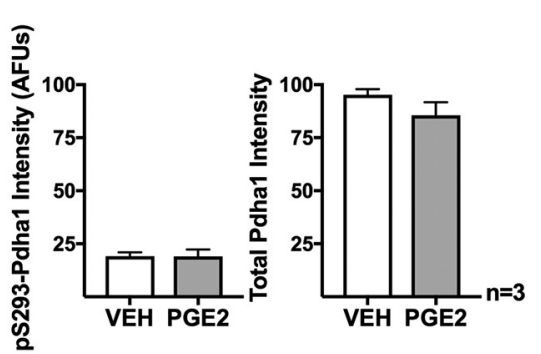

f.

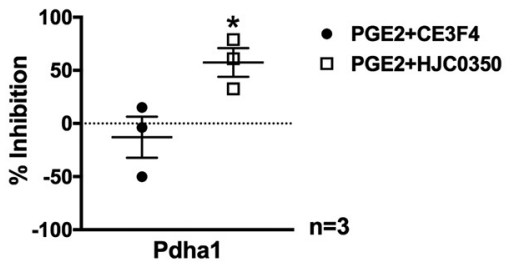

h.

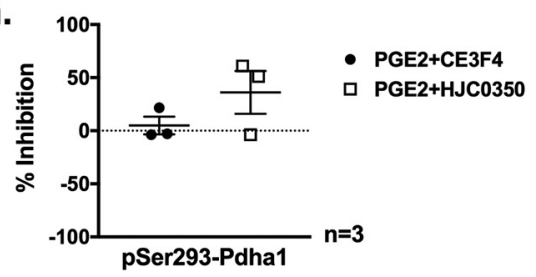

j.

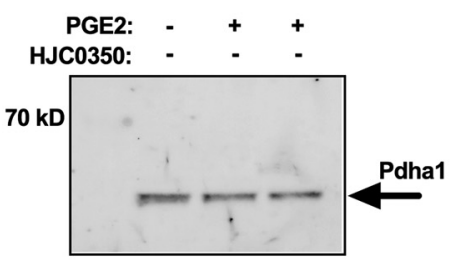

Figure 5. Systemic Epac2 inhibition prevents acute PGE2-induced heat hyperalgesia in male mice. $\boldsymbol{a}$, Mean paw withdrawal latencies from noxious heat after $1 \mathrm{ng} / 20 \mu \mathrm{l}$ PGE2 hindpaw injection with or without systemic Epac inhibitors ( $3 \mathrm{mg} / \mathrm{kg}$, i.p.) in male mice. Statistical significance was determined by two-way ANOVA with Sidak multiple-comparisons test; $* * * p<0.001$, $* * p<0.01$. $\boldsymbol{b}$, The band intensity of $p$-Pdha1 and the PKC p-substrate from L3-5 DRGs rapidly dissected immediately after in vivo PGE2 hindpaw administration and behavioral testing in $\boldsymbol{a}$. c, Western blot of pS293-Pdha1, Pdha1, and tubulin from total (unfractionated) DRG lysate collected from male mice $1 \mathrm{~h}$ after hindpaw injection of PGE2. $d$, Quantification of the intensity of bands in $\boldsymbol{c}(p=n s, n=3) . \boldsymbol{e}, \boldsymbol{g}$, Pdha1 $(\boldsymbol{e})$ and p-S293-Pdha1 $(\boldsymbol{g})$ Western blots of L3-5 DRG $1 \mathrm{~h}$ after hindpaw PGE2 injection. $\boldsymbol{e}-\boldsymbol{g}$, The scatter plots $(\boldsymbol{f}, \boldsymbol{h})$ quantify the percentage of inhibition of PGE2-induced p-Pdha1 (e) and p-S293-Pdha1 (g) signal intensity in phospho-enriched samples by CE3F4 (Epac1 inhibitor), or HJC0350 (Epac2 inhibitor). $\boldsymbol{i}$, Paw withdrawal latencies from noxious heat after hindpaw injection of $1 \mathrm{ng} / 20 \mu \mathrm{l}$ PGE2 with or without systemic HJC0350 administration (3 mg/kg, i.p.) in female mice. Statistical significance was determined by two- 
a. Hargreaves: Male

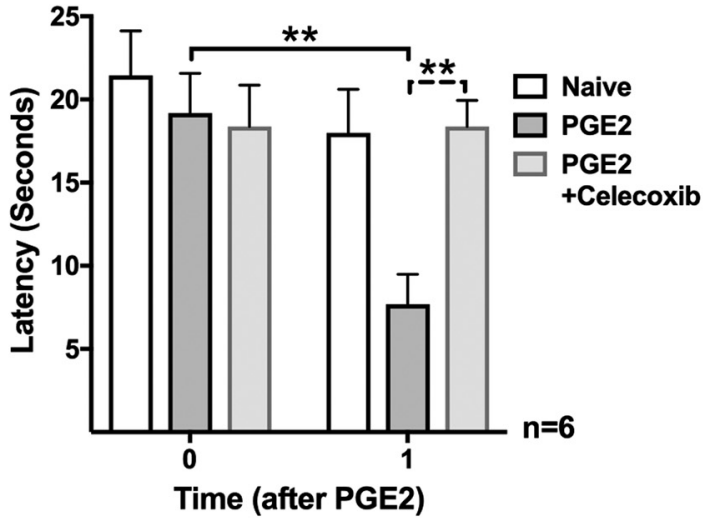

C.

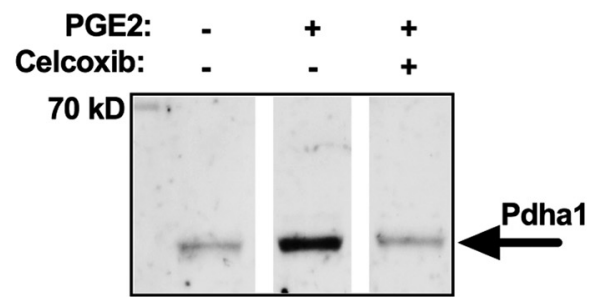

e.

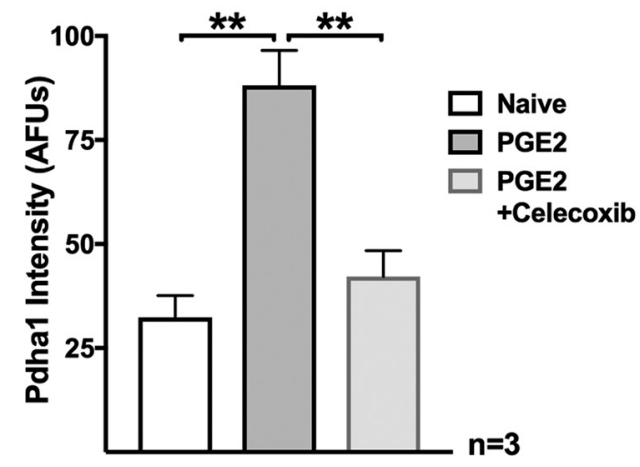

b.
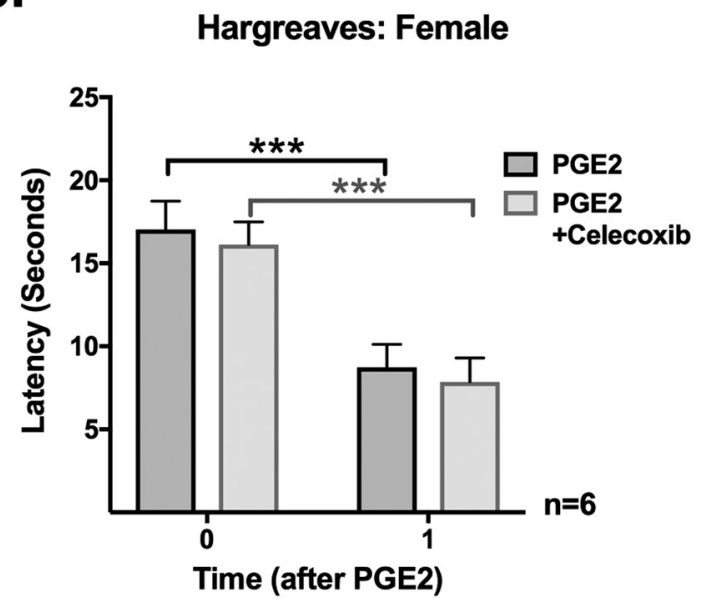

d.

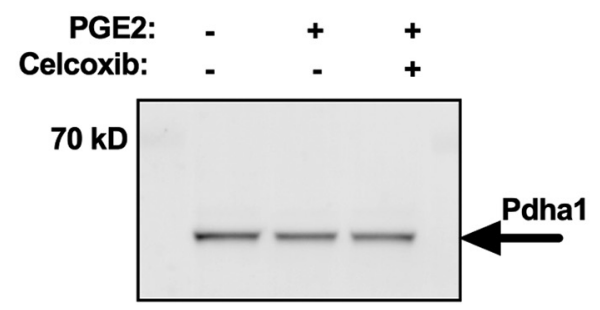

f.

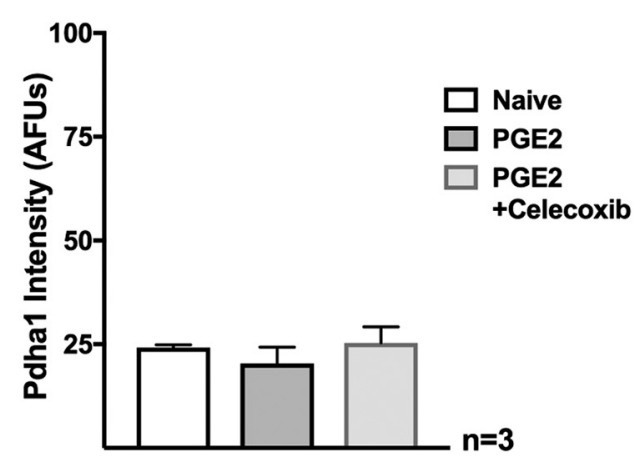

Figure 6. Induction of p-Pdha1 and hyperalgesia by hindpaw injection of PGE2 requires endogenous COX2 activity in male mice. $\boldsymbol{a}, \boldsymbol{b}$, Mean paw withdrawal latencies from noxious heat after hindpaw injection of $1 \mathrm{ng} / 20 \mu \mathrm{l}$ PGE2 with or without systemic administration of $30 \mathrm{mg} / \mathrm{kg}$ celecoxib, i.p., in male $(\boldsymbol{a})$ and female $(\boldsymbol{b})$ mice, $1 \mathrm{~h}$ after injection. Statistical significance was determined by two-way ANOVA with Sidak multiple-comparisons test; $* * p<0.01, * * * p<0.001$. $\boldsymbol{c}, \boldsymbol{d}$, Immediately after behavioral testing, L3-5 DRGs from male (c) and female (d) mice were dissected, and phospho-protein-enriched samples were prepared for Pdha1 Western blots ( $n=3,2$ mice pooled per sample). $\boldsymbol{e}, \boldsymbol{f}$, Pdha1 blot signal intensity was quantified for male (e) and female $(f)$ mice, and significance was determined by one-way ANOVA with Tukey's multiple-comparisons test; $* * p<0.01$.

innervating the injured tissue. Therefore, to determine whether endogenous prostaglandin synthesis is necessary for ganglionic p-Pdhal induced by noxious insult, we administered the cyclooxygenase 2 (COX2) inhibitor celecoxib $(30 \mathrm{mg} / \mathrm{kg}$, i.p.) before hindpaw injection of PGE2. Systemic celecoxib prevented PGE2induced heat hyperalgesia in male mice $(\mathrm{PGE} 2=7.69 \pm 1.8$; vs

way ANOVA with Sidak multiple-comparisons test; $* * * p<0.001 ; * * p<0.01 ; * p<0.05$. $\boldsymbol{j}$, Pdha1 Western blot of phospho-enriched samples from DRGs dissected immediately after behavioral testing in $\boldsymbol{i} . \boldsymbol{k}$, Quantification of Pdha1 band intensity from $\boldsymbol{j}$.
PGE2 + celecoxib $=18.38 \pm 1.6 ; p=0.0076$, two-way ANOVA, $n=6$; Fig. $6 a$ ), but it did not have a significant effect in females ( $p=\mathrm{ns}$, two-way ANOVA, $n=6$; Fig. $6 b$ ). Furthermore, treatment with celecoxib suppressed the induction of p-Pdha1 in L35 DRGs by hindpaw PGE2 injection $(p=0.0072$, two-way ANOVA, $n=3$ ) in male mice (Fig. $6 c, e$ ). The administration of PGE2 in female mice did not increase in p-Pdhal in DRGs, and p-Pdha1 levels were not affected by celecoxib (Fig. $6 d$,f), suggesting that mechanisms underlying PGE2-induced heat hyperalgesia diverge between male and female mice.

Enhancing mitochondrial respiration increases the mitochondrial membrane potential $(\Delta \Psi \mathrm{m})$ and the production of ATP 
a.

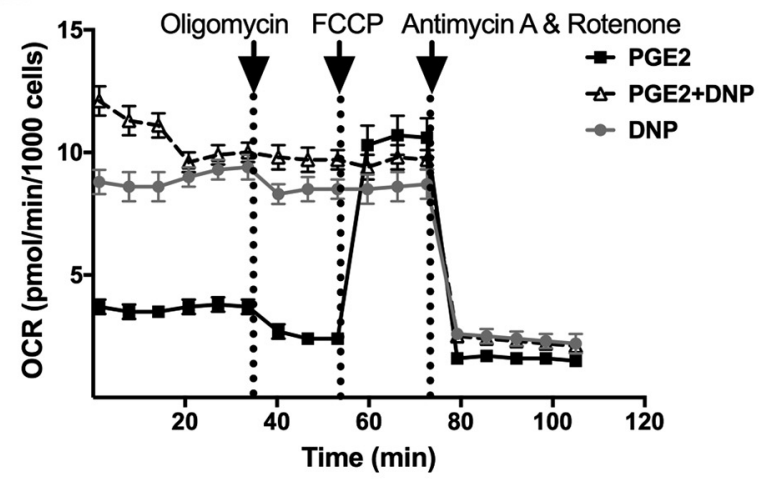

C.

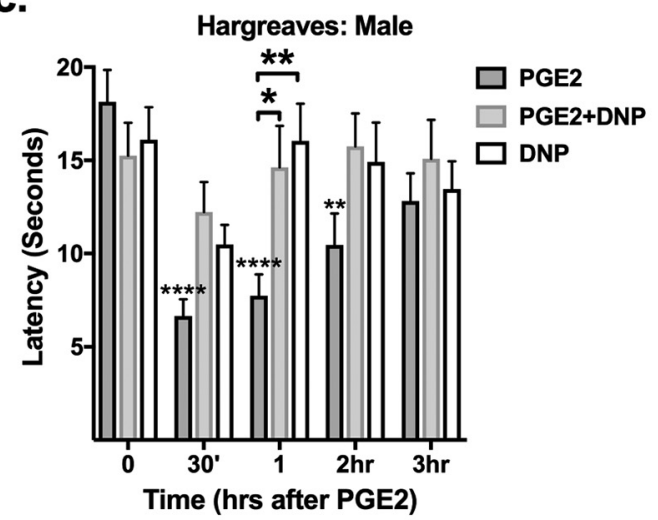

b.

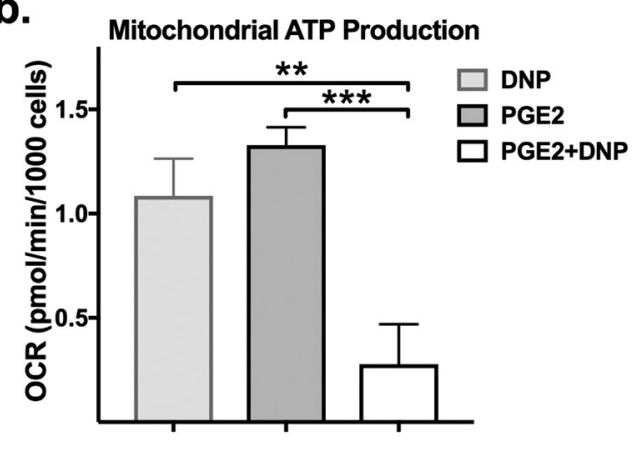

d.

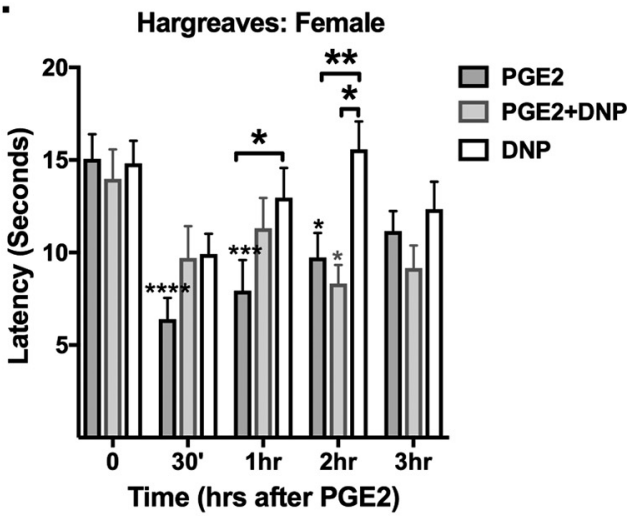

e.

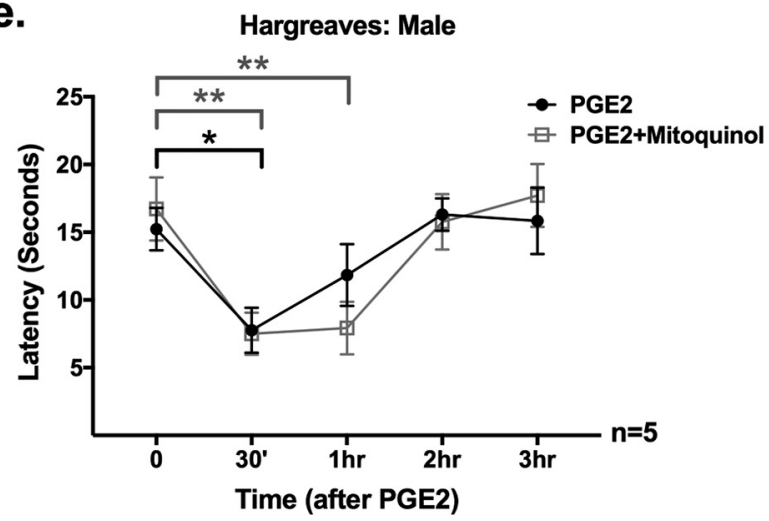

Figure 7. DNP prevents PGE2-induced heat hyperalgesia in males. $\boldsymbol{a}$, The OCR from dissociated DRGs stimulated with $10 \mu \mathrm{M}$ PGE2 with or without $100 \mu \mathrm{m}$ DNP (mild mitochondrial uncoupler) in the presence of Mito Stress Test drugs. $\boldsymbol{b}$, Quantification of OCR attributable to ATP production for PGE2-stimulated DRG cells with or without $100 \mu \mathrm{m}$ DNP. Statistical significance was determined by ordinary one-way ANOVA with Tukey's multiple-comparisons test ( $* * p<0.01 ; * * * p<0.001$; technical replicates: DNP $=5, P G E 2=9, P G E 2+D N P=7$ ). $\boldsymbol{c}, \boldsymbol{d}$, Mean paw withdrawal latencies from noxious heat after $1 \mathrm{ng} / 20 \mu \mathrm{l}$ PGE2 hindpaw injection with or without systemic administration of $5 \mathrm{mg} / \mathrm{kg}$ DNP in male (c) and female (d) mice. Significance denoted by asterisks without a comparison bracket is compared with time 0 for that same group. Asterisks above a comparison bracket compare two different groups at the same time point. $\boldsymbol{e}$, Mean paw withdrawal latencies from noxious heat after $1 \mathrm{ng} / 20 \mu \mathrm{l}$ PGE2 hindpaw injection with or without systemic $20 \mathrm{mg} / \mathrm{kg}$ mitoquinol injection in male C57BL/6J mice. Statistical significance was determined by two-way ANOVA with Tukey's multiple-comparisons test $(* p<0.05, * * p<0.01, * * * p<0.001, * * * * p<0.0001)$.

and ROS. Inhibition of Epac2 significantly suppressed the increase in Pdhal phosphorylation and mitochondrial respiration in vitro, as well as the heat hyperalgesia produced by PGE2 in males in vivo. To explore the possibility that changes in mitochondrial function contribute to acute heat hyperalgesia, we first applied the $\Delta \Psi \mathrm{m}$ uncoupler DNP $(100 \mu \mathrm{M})$ to dissociated DRG cells from male mice in the presence of PGE2. DNP significantly reduced OCR attributable to ATP production from $1.33 \pm 0.09$ to $0.28 \pm 0.19$ OCR $(p=0.0001$, one-way ANOVA; five to nine replicates each; Fig. $7 a, b)$. Next, we administered DNP $(5 \mathrm{mg} / \mathrm{kg}$, i.p.) in vivo, a low dose reported to be nontoxic in vivo (Liu et al., 2015). Strikingly, systemic DNP administration prevented PGE2- induced heat hyperalgesia in male mice $(1 \mathrm{~h}$ PGE $2=7.74 \pm 1.14$ compared with 1 h PGE2 $+\mathrm{DNP}=14.63 \pm 2.22 ; p=0.0129$, twoway ANOVA, $n=6$; Fig. $7 c$ ), but not in female mice ( $p=$ ns, twoway ANOVA, $n=6$; Fig. $7 d$ ), similar to the sex difference seen with HJC0350 and celecoxib. Since DNP prevented hyperalgesia in males, we next evaluated whether ROS production, a byproduct of mitochondrial respiration, contributes to acute PGE2-induced heat hyperalgesia. Although ROS scavengers have been shown to relieve persistent pain (Schwartz et al., 2009), intraperitoneal administration of the mitochondria-targeted ROS scavenger mitoquinol $(20 \mathrm{mg} / \mathrm{kg})$ did not prevent acute PGE2-induced heat hyperalgesia in male mice (Fig. 7e). This experiment provides initial evidence 


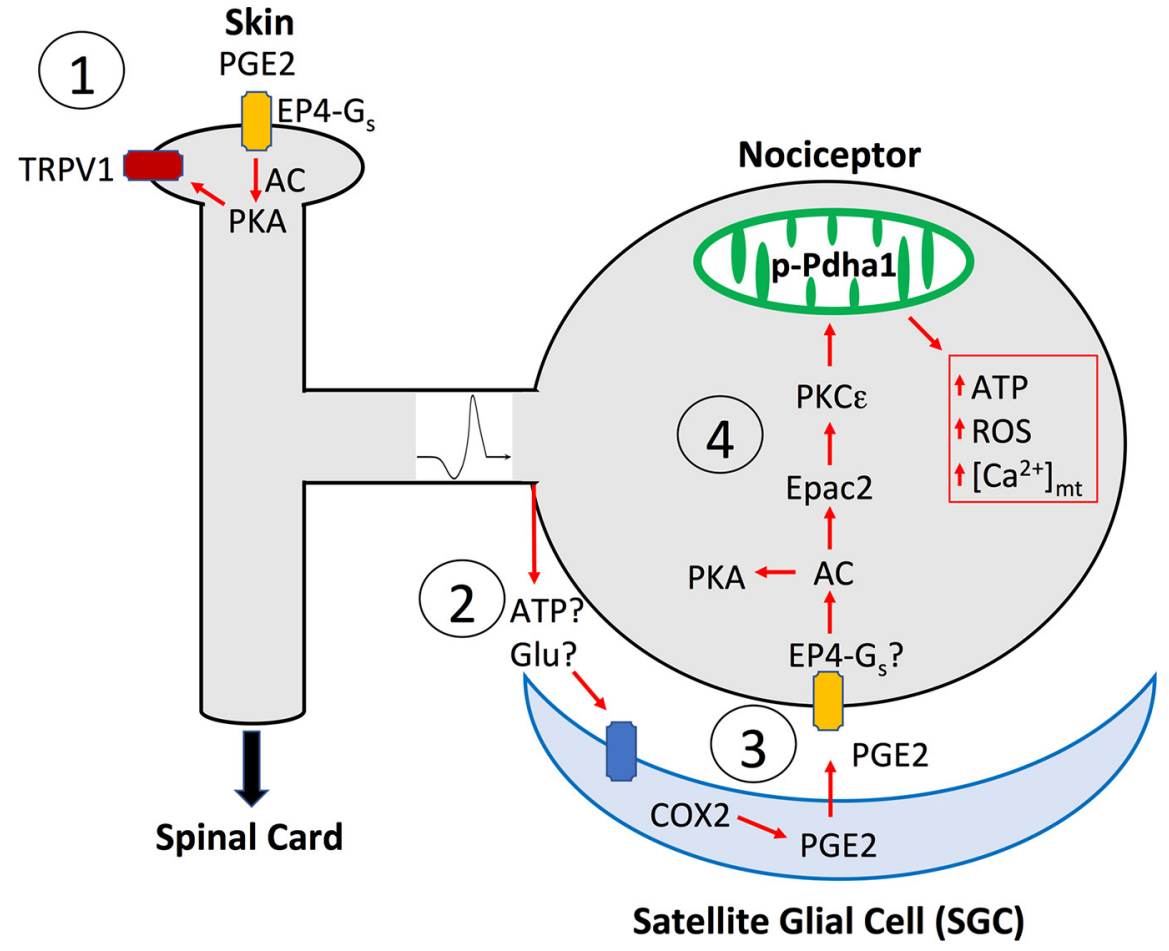

Figure 8. Model: $G_{s}-E p a c$ signaling acutely regulates Pdha1 to enhance mitochondrial function and promote heat hyperalgesia in male mice. This cartoon presents a model for the in vivo regulation of mitochondrial function by adenylyl cyclase signaling in sensory neurons, as follows. 1, Local hindpaw injection of PGE2 acts via $\mathrm{G}_{\mathrm{s}}$-coupled receptors and PKA to sensitize axon terminals, as previously reported (Sugden and Holness, 2011). 2, Nociceptor action potential firing causes neuronal cell bodies to release messengers within the DRG that stimulate the production of arachidonic acid, the substrate for COX enzymes, within non-neuronal cells such as satellite glial cells. The nature of these messengers remains to be determined, but glutamate (Ferrari et al., 2014) and ATP (Rozanski et al., 2013) have been implicated in the literature. 3, Intraganglionic COX2 activity leads to local synthesis and release of PGE2, which binds to $\mathrm{G}_{s}$-coupled prostaglandin receptors (4), most likely EP4, on DRG neurons to activate Epac2. Epac2 and PKC $\varepsilon$ are widely expressed in naive DRG neurons and are required for the phosphorylation of Pdha1 at a site or sites distinct from PDK substrate residues. Epac2-induced phosphorylation of Pdha1 enhances mitochondrial function in affected neurons. Signaling illustrated in 4 occurs in dissociated neurons from both male and female mice, but phosphorylation of Pdha1 in vivo in response to hindpaw injection of PGE2 occurs only in males. Systemic administration of the mitochondrial uncoupler DNP protected male but not female mice against PGE2-induced heat hyperalgesia, supporting the conclusion that mitochondrial function contributes to acute inflammatory hyperalgesia. Further investigation will be required to fully corroborate this model.

that ROS are not responsible for Epac2-dependent hyperalgesia, but additional experiments will be necessary to definitively rule out a role for ROS and reactive nitrogen species (Grace et al., 2016).

Collectively, these results reveal a novel mechanism for the positive regulation of Pdhal and mitochondrial respiration by Epac2-PKC $\varepsilon$ signaling in response to an acute inflammatory insult in male mice. We propose a model for this process in which nociceptor activation leads to intraganglionic prostaglandin release from SGCs. Prostaglandins then act through receptors on neuronal cell bodies to activate Epac2, which enhances Pdhal function, mitochondrial respiration, and ATP production (Fig. 8).

\section{Discussion}

Pdhal is a target of Epac-PKC signaling in vitro

Epac signaling has been implicated in a variety of chronic pain models, but is generally considered dispensable for acute nociceptor sensitization (Reichling and Levine, 2009). Most studies focus on Epac1, which is upregulated after injury and appears to act primarily in nonpeptidergic, IB4 (isolectin B4)-binding sensory neurons to enhance nociception (Hucho et al., 2005;
Eijkelkamp et al., 2010). Epac2-PKC $\varepsilon$ signaling reportedly participates in nociceptor sensitization through the enhancement of calcitonin gene-related peptide release and TRPV1 function; like Epac1, it is upregulated in response to inflammatory injury (Vasko et al., 2014; Shariati et al., 2016; Gu et al., 2016a). Two targets of Epac-PKC $\varepsilon$ signaling have been proposed to mediate mechanical hypersensitivity during inflammation, Piezo-2 (Singhmar et al., 2016) and P2X3 (Wang et al., 2007); however, neither channel is extensively expressed in murine TRPV1-expressing nociceptors (Zwick et al., 2002; Cavanaugh et al., 2009; Usoskin et al., 2015).

cAMP plays important roles in nociceptor sensitization mediating inflammatory hyperalgesia. PKA is generally considered to be the key effector of cAMP in this process, sensitizing nociceptors by phosphorylating and enhancing the function of multiple ion channels in peripheral terminals (Taiwo et al., 1989; Aley and Levine, 1999). In our experiments, we found that PGE2 acutely activates Epac and PKC in dissociated DRG neurons. To identify proteins phosphorylated by $\mathrm{PKC}$ in response to Epac activation, we isolated total phospho-protein from dissociated DRGs by IMAC, followed by Western blot analysis using an antibody that recognizes phospho-serine substrates of PKC (Goode and Molliver, 2019). We restricted our analysis to Epac-dependent phospho-proteins by comparing PGE2 stimulation in the presence or absence of Epac inhibitors. We identified the most prominent band as Pdhal by LC-MS/MS and confirmed the target identity by demonstrating Epac-dependent recruitment of Pdhal to phospho-protein fractions. Pdhal phosphorylation was visualized within $30 \mathrm{~s}$ of PGE2 application and was prevented by the inhibition of either Epac or $\mathrm{PKC} \varepsilon$, which is expressed in most DRG neurons (Khasar et al., 1999). In contrast, total Pdhal protein levels did not change in response to PGE2 in vitro or in vivo.

Pdhal links glucose metabolism with mitochondrial respiration by catalyzing the irreversible oxidation of pyruvate, produced from cytoplasmic glucose, to acetyl-CoA. Acetyl-CoA initiates the TCA cycle, producing nicotinamide adenine dinucleotide and flavin adenine dinucleotide, which pass electrons to complex I and II of the electron transport chain, respectively. As a result, increasing Pdhal activity increases the $\Delta \Psi \mathrm{m}$, which provides the electrochemical gradient required for the production of ATP. The $\Delta \Psi \mathrm{m}$ also allows mitochondria to buffer cytosolic $\mathrm{Ca}^{2+}$ levels by importing $\mathrm{Ca}^{2+}$ (Nicholls and Ward, 2000; Shutov et al., 2013).

Inhibitory phosphorylation of Pdha1 by PDK and its reversal by PDP is the principal established mechanism for the regulation of Pdhal function (Gray et al., 2014). Loss-of-function mutations in Pdhal lead to systemic lactic acidosis, and the PDK inhibitor DCA has been used clinically to promote mitochondrial respiration in congenital disorders of oxidative phosphorylation such as 
Leigh syndrome, which can lead to encephalomyelopathy and peripheral neuropathy (Kimura et al., 1997; Koga et al., 2012). In contrast to the phosphorylation of Pdhal induced by acetyl-CoA application to activate PDK, phosphorylation of Pdhal induced by PGE2 was insensitive to inhibition by DCA, did not increase p-S293, and resulted in enhanced mitochondrial respiration, a result consistent with increased availability of the Pdha1 product acetyl-CoA. These findings indicate that Epac2 signaling does not activate PDK, but instead positively modulates Pdhal function through phosphorylation of a noncanonical serine residue yet to be identified. Several Pdhal phospho-serines that are not PDK substrates have been identified in mouse tissues (Huttlin et al., 2010), but have not been functionally characterized, and we were unable to find any previous reports describing positive regulation of Pdhal mediated by phosphorylation. The reduction of mitochondrial respiration by PDK-mediated inhibition of Pdhal was recently proposed to underlie chemotherapy-induced neuropathic pain (Ludman and Melemedjian, 2019). Those findings, combined with our results, suggest that there are profound changes in the regulation of mitochondrial function that occur during the transition from acute to chronic pain, and that Pdhal may represent an important locus for those changes. It will be exciting to determine whether enhancement of Pdhal function by Epac2 can be exploited therapeutically to counteract reduced Pdha1 function in neuropathic pain conditions or in other metabolic disorders such as Leigh syndrome.

\section{PGE2-induced hyperalgesia and Pdhal phosphorylation in vivo}

As expected, hindpaw injection of PGE2 produced hyperalgesia lasting several hours that was blocked by inhibition of PKA. Systemic administration of HJC0350, but not CE3F4, suppressed both hyperalgesia and Pdhal phosphorylation in male mice, establishing a role for Epac2 in acute inflammatory hyperalgesia. From these results, we conclude that both PKA and Epac2 are required for the full manifestation of hyperalgesia. Given the rapid induction of p-Pdhal within the DRG in response to a distant insult, we hypothesized that nociceptor firing was driving ganglionic Pdhal phosphorylation. SGCs within the DRG reportedly synthesize PGE2 in response to noxious stimulation (Capuano et al., 2009; Souza et al., 2013). To test a role for prostaglandin synthesis in acute hyperalgesia, celecoxib was given systemically before hindpaw PGE2 injection. Celecoxib attenuated PGE2-induced hyperalgesia and ganglionic p-Pdha1 in male mice, supporting the interpretation that Epac2 activation in DRGs in vivo requires local prostaglandin synthesis. Lack of mRNA for COX1 or COX2 in naive isolated sensory neurons argues against an autocrine mechanism (Usoskin et al., 2015). However, both transcripts are present in whole DRGs (Goswami et al., 2014; Usoskin et al., 2015; Sapio et al., 2016), which is consistent with the above reports of prostaglandin synthesis by SGCs. Neuronal activity-dependent prostaglandin release within the DRG provides a possible explanation for the rapid phosphorylation of Pdhal in DRGs in response to peripheral insult, which is supported by a growing body of evidence that SGCs participate in nociceptor sensitization and hyperalgesia (Hanani, 2005; Souza et al., 2013; Ferrari et al., 2014). Given that PKA was also required for hyperalgesia, and that PKA is known to enhance nociceptive transduction in peripheral terminals, we propose a model in which PKA is necessary for the initial nociceptor sensitization and firing required to induce prostaglandin synthesis within the DRG, which then leads to ganglionic Pdha1 phosphorylation (Fig. 8). Although the cell body is often considered unimportant for action potential propagation across the axonal $\mathrm{T}$-junction to the central synapse, there is compelling evidence that the cell body contributes to nociceptive transmission (Du et al., 2014).

To test the impact of mitochondrial function on acute hyperalgesia, we administered the $\triangle \Psi \mathrm{M}$ uncoupler DNP in vivo. DNP attenuated PGE2-induced hyperalgesia, which is consistent with a role for Epac2-induced mitochondrial hyperpolarization in hyperalgesia. Although perhaps counterintuitive, neuroprotective effects of DNP have been reported, including prevention of sciatic nerve degeneration following crush injury (da Costa et al., 2010; Geisler et al., 2017). In addition to producing ATP, mitochondria participate in cell signaling through the production of $\mathrm{NAD}^{+}$and ROS, $\mathrm{Ca}^{2+}$ buffering, fatty acid and amino acid synthesis, translational regulation, and cellular stress responses (Chandel, 2015). Most investigation of mitochondrial contributions to chronic pain has focused on mitochondrial dysfunction or production of ROS (Flatters, 2015). Our results suggest that increased mitochondrial respiration contributes to nociceptive signaling and scavenging of ROS with the mitochondria-targeted antioxidant mitoquinol was not sufficient to prevent PGE2 hyperalgesia. Mitochondrial regulation of calcium signaling may be highly significant for enhanced nociceptive transmission during mitochondrial hyperpolarization, as calcium uptake is dependent on the $\Delta \Psi \mathrm{M}$. Following calcium uptake, the prolonged release of calcium by mitochondria has been reported to increase presynaptic glutamate release and central sensitization at the spinal synapse, and likely contributes to activity-dependent regulation of both transcription and translation (Flatters, 2015).

\section{A possible sex-specific role for satellite glial cells in the regulation of sensory neuron mitochondrial function}

We found that PGE2 rapidly stimulated the phosphorylation of Pdha1, but not at PDK substrate residue S293, in dissociated neurons from both male and female mice. Hindpaw injection of PGE2 stimulated phosphorylation of Pdha1, but only in males, although both sexes displayed hyperalgesia. Likewise, the inhibition of Epac2 or COX2 reversed PGE2-induced hyperalgesia in males, but not in females. These results suggest that PGE2-Epac2Pdhal signaling is intact in female DRG neurons, but that the induction of local ganglionic prostaglandin signaling by a distal insult does not occur. In a perhaps related report, the NSAID ibuprofen similarly provided analgesia in an acute pain model in male but not female human volunteers (Walker and Carmody, 1998). A recent transcriptomic analysis also found sex differences in the expression of prostaglandin signaling components, as well as enhanced hyperalgesia in females in response to higher doses of PGE2 than used here (Tavares-Ferreira et al., 2020).

Collectively, this work demonstrates a novel mechanism enhancing mitochondrial function through Epac2-PKC $\varepsilon$ signaling. Although Epac2 signaling promoted hyperalgesia, the activation of neuronal Epac2 by paracrine prostaglandins may serve a homeostatic role, protecting neurons from mitochondrial depolarization and ATP depletion resulting from the energetic stress of persistent firing. In a broader context, the regulation of Pdha1 function by Epac2-PKC may be a common receptor-mediated mechanism in cells expressing Epac2, although we have not yet explored this mechanism in other tissues.

\section{References}

Aley KO, Levine JD (1999) Role of protein kinase A in the maintenance of inflammatory pain. J Neurosci 19:2181-2186. 
Aley KO, Messing RO, Mochly-Rosen D, Levine JD (2000) Chronic hypersensitivity for inflammatory nociceptor sensitization mediated by the epsilon isozyme of protein kinase C. J Neurosci 20:4680-4685.

Almahariq M, Tsalkova T, Mei FC, Chen H, Zhou J, Sastry SK, Schwede F, Cheng X (2013) A novel EPAC-specific inhibitor suppresses pancreatic cancer cell migration and invasion. Mol Pharmacol 83:122-128.

Bhave G, Zhu W, Wang H, Brasier DJ, Oxford GS, Gereau RW 4th (2002) cAMP-dependent protein kinase regulates desensitization of the capsaicin receptor (VR1) by direct phosphorylation. Neuron 35:721-731.

Bonnet S, Archer SL, Allalunis-Turner J, Haromy A, Beaulieu C, Thompson R, Lee CT, Lopaschuk GD, Puttagunta L, Bonnet S, Harry G, Hashimoto K, Porter CJ, Andrade MA, Thebaud B, Michelakis ED (2007) A mitochondria- $\mathrm{K}+$ channel axis is suppressed in cancer and its normalization promotes apoptosis and inhibits cancer growth. Cancer Cell 11:37-51.

Caldeira da Silva CC, Cerqueira FM, Barbosa LF, Medeiros MHG, Kowaltowski AJ (2008) Mild mitochondrial uncoupling in mice affects energy metabolism, redox balance and longevity. Aging Cell 7:552-560.

Candiano G, Bruschi M, Musante L, Santucci L, Ghiggeri GM, Carnemolla B, Orecchia P, Zardi L, Righetti PG (2004) Blue silver: a very sensitive colloidal Coomassie G-250 staining for proteome analysis. Electrophoresis 25:1327-1333.

Capuano A, De Corato A, Lisi L, Tringali G, Navarra P, Dello Russo C (2009) Proinflammatory-activated trigeminal satellite cells promote neuronal sensitization: relevance for migraine pathology. Mol Pain 5:43.

Cavanaugh DJ, Lee H, Lo L, Shields SD, Zylka MJ, Basbaum AI, Anderson DJ (2009) Distinct subsets of unmyelinated primary sensory fibers mediate behavioral responses to noxious thermal and mechanical stimuli. Proc Natl Acad Sci U S A 106:9075-9080.

Chandel NS (2015) Evolution of mitochondria as signaling organelles. Cell Metab 22:204-206.

Christie K, Koshy D, Cheng C, Guo G, Martinez JA, Duraikannu A, Zochodne DW (2015) Intraganglionic interactions between satellite cells and adult sensory neurons. Mol Cell Neurosci 67:1-12.

Cook KG, Bradford AP, Yeaman SJ, Aitken A, Fearnley IM, Walker JE (1984) Regulation of bovine kidney branched-chain 2-oxoacid dehydrogenase complex by reversible phosphorylation. Eur J Biochem 145:587591.

da Costa RFM, Martinez AMB, Ferreira ST (2010) 2,4-Dinitrophenol blocks neurodegeneration and preserves sciatic nerve function after trauma. J Neurotrauma 27:829-841.

Du X, Hao H, Gigout S, Huang D, Yang Y, Li L, Wang C, Sundt D, Jaffe DB, Zhang H, Gamper N (2014) Control of somatic membrane potential in nociceptive neurons and its implications for peripheral nociceptive transmission. Pain 155:2306-2322.

Eijkelkamp N, Wang H, Garza-Carbajal A, Willemen HLDM, Zwartkruis FJ, Wood JN, Dantzer R, Kelley KW, Heijnen CJ, Kavelaars A (2010) Low nociceptor GRK2 prolongs prostaglandin E2 hyperalgesia via biased cAMP signaling to Epac/Rap1, protein kinase $\mathrm{C} \varepsilon$, and MEK/ERK. J Neurosci 30:12806-12815.

Eijkelkamp N, Linley JE, Torres JM, Bee L, Dickenson AH, Gringhuis M, Minett MS, Hong GS, Lee E, Oh U, Ishikawa Y, Zwartkuis FJ, Cox JJ, Wood JN (2013) A role for Piezo2 in EPAC1-dependent mechanical allodynia. Nat Commun 4:1682.

England S, Bevan S, Docherty RJ (1996) PGE2 modulates the tetrodotoxinresistant sodium current in neonatal rat dorsal root ganglion neurones via the cyclic AMP-protein kinase A cascade. J Physiol 495:429-440.

Ferrari LF, Lotufo CM, Araldi D, Rodrigues MA, Macedo LP, Ferreira SH, Parada CA (2014) Inflammatory sensitization of nociceptors depends on activation of NMDA receptors in DRG satellite cells. Proc Natl Acad Sci U S A 111:18363-18368.

Ferreira SH, Nakamura M (1979) I - Prostaglandin hyperalgesia, a cAMP/ $\mathrm{Ca} 2+$ dependent process. Prostaglandins 18:179-190.

Feuerstein I, Morandell S, Stecher G, Huck CW, Stasyk T, Huang H-L, Huber LA, Bonn GK (2005) Phosphoproteomic analysis using immobilized metal ion affinity chromatography on the basis of cellulose powder. Proteomics 5:46-54.

Fitzgerald EM, Okuse K, Wood JN, Dolphin AC, Moss SJ (1999) cAMP-dependent phosphorylation of the tetrodotoxin-resistant voltage-dependent sodium channel SNS. J Physiol 516:433-446.

Flatters SJL (2015) The contribution of mitochondria to sensory processing and pain. Prog Mol Biol Transl Sci 131:119-146.
Fujita T, Umemura M, Yokoyama U, Okumura S, Ishikawa Y (2017) The role of Epac in the heart. Cell Mol Life Sci 74:591-606.

Geisler JG, Marosi K, Halpern J, Mattson MP (2017) DNP, mitochondrial uncoupling, and neuroprotection: a little dab'll do ya. Alzheimers Dement 13:582-591.

Goode D, Molliver DC (2019) Phospho-substrate profiling of Epac-dependent protein kinase $\mathrm{C}$ activity. Mol Cell Biochem 456:167-178.

Goswami SC, Mishra SK, Maric D, Kaszas K, Gonnella GL, Clokie SJ, Kominsky HD, Gross JR, Keller JM, Mannes AJ, Hoon MA, Iadarola MJ (2014) Molecular signatures of mouse TRPV1-lineage neurons revealed by RNA-Seq transcriptome analysis. J Pain 15:1338-1359.

Grace PM, Gaudet AD, Staikopoulos V, Maier SF, Hutchinson MR, Salvemini D, Watkins LR (2016) Nitroxidative signaling mechanisms in pathological pain. Trends Neurosci 39:862-879.

Gray LR, Tompkins SC, Taylor EB (2014) Regulation of pyruvate metabolism and human disease. Cell Mol Life Sci 71:2577-2604.

Gu Y, Li G, Chen Y, Huang L-YM (2016a) Epac-protein kinase C alpha signaling in purinergic P2X3R-mediated hyperalgesia after inflammation. Pain 157:1541-1550.

Gu Y, Wang C, Li G, Huang L-YM (2016b) F-actin links Epac-PKC signaling to purinergic $\mathrm{P} 2 \mathrm{X} 3$ receptor sensitization in dorsal root ganglia following inflammation. Mol Pain 12:1-11.

Gu Y, Li G, Huang L-YM (2018) Inflammation induces Epac-protein kinase $\mathrm{C}$ alpha and epsilon signaling in TRPV1-mediated hyperalgesia. Pain 159:2383-2393.

Hanani M (2005) Satellite glial cells in sensory ganglia: from form to function. Brain Res Brain Res Rev 48:457-476.

Hanani M (2012) Intercellular communication in sensory ganglia by purinergic receptors and gap junctions: implications for chronic pain. Brain Res 1487:183-191.

Hingtgen CM, Waite KJ, Vasko MR (1995) Prostaglandins facilitate peptide release from rat sensory neurons by activating the adenosine $3^{\prime}, 5^{\prime}$-cyclic monophosphate transduction cascade. J Neurosci 15:5411-5419.

Huang B, Gudi R, Wu P, Harris R, Hamilton J, Popov K (1998) Isoenzymes of pyruvate dehydrogenase phosphatase. DNA-derived amino acid sequences, expression, and regulation. J Biol Chem 273:17680-17688.

Hucho TB, Dina OA, Levine JD (2005) Epac mediates a cAMP-to-PKC signaling in inflammatory pain: an isolectin B4(+) neuron-specific mechanism. J Neurosci 25:6119-6126.

Huttlin EL, Jedrychowski MP, Elias JE, Goswami T, Rad R, Beausoleil SA, Villen J, Haas W, Sowa ME, Gygi SP (2010) A tissue-specific atlas of mouse protein phosphorylation and expression. Cell 143:1174-1189.

Kase H, Iwahashi K, Nakanishi S, Matsuda Y, Yamada K, Takahashi M, Murakata C, Sato A, Kaneko M (1987) K-252 compounds, novel and potent inhibitors of protein kinase $\mathrm{C}$ and cyclic nucleotide-dependent protein kinases. Biochem Biophys Res Commun 142:436-440.

Kashima Y, Miki T, Shibasaki T, Ozaki N, Miyazaki M, Yano H, Seino S (2001) Critical role of cAMP-GEFII-Rim2 complex in incretin-potentiated insulin secretion. J Biol Chem 276:46046-46053.

Kerbey AL, Randle PJ, Cooper RH, Whitehouse S, Pask HT, Denton RM (1976) Regulation of pyruvate dehydrogenase in rat heart. Mechanism of regulation of proportions of dephosphorylated and phosphorylated enzyme by oxidation of fatty acids and ketone bodies and of effects of diabetes: role of coenzyme A, acetyl-coenzyme A and reduced and oxidized nicotinamide-adenine dinucleotide. Biochem J 154:327-348.

Khasar SG, Lin YH, Martin A, Dadgar J, McMahon T, Wang D, Hundle B, Aley KO, Isenberg W, McCarter G, Green PG, Hodge CW, Levine JD, Messing RO (1999) A novel nociceptor signaling pathway revealed in protein kinase C epsilon mutant mice. Neuron 24:253-260.

Kimura S, Ohtuki N, Nezu A, Tanaka M, Takeshita S (1997) Clinical and radiologic improvements in mitochondrial encephalomyelopathy following sodium dichloroacetate therapy. Brain Dev 19:535-540.

Koga Y, Povalko N, Katayama K, Kakimoto N, Matsuishi T, Naito E, Tanaka M (2012) Beneficial effect of pyruvate therapy on Leigh syndrome due to a novel mutation in PDH E1 $\alpha$ gene. Brain Dev 34:87-91.

Lin C-R, Amaya F, Barrett L, Wang H, Takada J, Samad TA, Woolf CJ (2006) Prostaglandin E2 receptor EP4 contributes to inflammatory pain hypersensitivity. J Pharmacol Exp Ther 319:1096-1103.

Linn TC, Pettit FH, Reed LJ (1969) Alpha-keto acid dehydrogenase complexes. X. Regulation of the activity of the pyruvate dehydrogenase complex from beef kidney mitochondria by phosphorylation and dephosphorylation. Proc Natl Acad Sci U S A 62:234-241. 
Liu D, Zhang Y, Gharavi R, Park HR, Lee J, Siddiqui S, Telljohann R, Nassar MR, Cutler RG, Becker KG, Mattson MP (2015) The mitochondrial uncoupler DNP triggers brain cell mTOR signaling network reprogramming and CREB pathway up-regulation. J Neurochem 134:677-692.

Ludman T, Melemedjian OK (2019) Bortezomib-induced aerobic glycolysis contributes to chemotherapy-induced painful peripheral neuropathy. Mol Pain 15:1744806919837429.

Malin SA, Davis BM, Molliver DC (2007) Production of dissociated sensory neuron cultures and considerations for their use in studying neuronal function and plasticity. Nat Protoc 2:152-160.

Medvedeva YV, Kim M-S, Usachev YM (2008) Mechanisms of prolonged presynaptic $\mathrm{Ca}^{2+}$ signaling and glutamate release induced by TRPV1 activation in rat sensory neurons. J Neurosci 28:5295-5311.

Meents JE, Fischer MJM, McNaughton PA (2017) Sensitization of TRPA1 by protein kinase A. PLoS One 12:e0170097.

Nesvizhskii AI, Keller A, Kolker E, Aebersold R (2003) A statistical model for identifying proteins by tandem mass spectrometry. Anal Chem 75:46464658.

Nicholls DG, Ward MW (2000) Mitochondrial membrane potential and neuronal glutamate excitotoxicity: mortality and millivolts. Trends Neurosci 23:166-174.

Nishikawa K, Toker A, Johannes FJ, Songyang Z, Cantley LC (1997) Determination of the specific substrate sequence motifs of protein kinase C isozymes. J Biol Chem 272:952-960.

Nita II, Caspi Y, Gudes S, Fishman D, Lev S, Hersfinkel M, Sekler I, Binshtok AM (2016) Privileged crosstalk between TRPV1 channels and mitochondrial calcium shuttling machinery controls nociception. Biochim Biophys Acta 1863:2868-2880.

Parada CA, Yeh JJ, Reichling DB, Levine JD (2003) Transient attenuation of protein kinase $\mathrm{C} \epsilon$ can terminate a chronic hyperalgesic state in the rat. Neuroscience 120:219-226.

Paulose-Ram R, Hirsch R, Dillon C, Losonczy K, Cooper M, Ostchega Y (2003) Prescription and non-prescription analgesic use among the US adult population: results from the third National Health and Nutrition Examination Survey (NHANES III). Pharmacoepidemiol Drug Saf 12:315-326.

Paxton R, Kuntz M, Harris RA (1986) Phosphorylation sites and inactivation of branched-chain alpha-ketoacid dehydrogenase isolated from rat heart, bovine kidney, and rabbit liver, kidney, heart, brain, and skeletal muscle. Arch Biochem Biophys 244:187-201.

Reichling DB, Levine JD (2009) Critical role of nociceptor plasticity in chronic pain. Trends Neurosci 32:611-618.

Reiner DJ, Lundquist EA (2018) Small GTPases. WormBook 2018:1-65.

Rozanski GM, Li Q, Kim H, Stanley EF (2013) Purinergic transmission and transglial signaling between neuron somata in the dorsal root ganglion. Eur J Neurosci 37:359-365.

Sapio MR, Goswami SC, Gross JR, Mannes AJ, Iadarola MJ (2016) Transcriptomic analyses of genes and tissues in inherited sensory neuropathies. Exp Neurol 283:375-395.

Schwartz ES, Kim HY, Wang J, Lee I, Klann E, Chung JM, Chung K (2009) Persistent pain is dependent on spinal mitochondrial antioxidant levels. J Neurosci 29:159-168.
Shariati B, Thompson EL, Nicol GD, Vasko MR (2016) Epac activation sensitizes rat sensory neurons through activation of Ras. Mol Cell Neurosci 70:54-67.

Shutov LP, Kim M-S, Houlihan PR, Medvedeva YV, Usachev YM (2013) Mitochondria and plasma membrane Ca2+-ATPase control presynaptic $\mathrm{Ca} 2+$ clearance in capsaicin-sensitive rat sensory neurons. J Physiol 591:2443-2462.

Singhmar P, Huo X, Eijkelkamp N, Berciano SR, Baameur F, Mei FC, Zhu Y, Cheng X, Hawke D, Mayor FJ, Murga C, Heijnen CJ, Kavelaars A (2016) Critical role for Epac1 in inflammatory pain controlled by GRK2-mediated phosphorylation of Epac1. Proc Natl Acad Sci U S A 113:3036-3041.

Souza GR, Talbot J, Lotufo CM, Cunha FQ, Cunha TM, Ferreira SH (2013) Fractalkine mediates inflammatory pain through activation of satellite glial cells. Proc Natl Acad Sci U S A 110:11193-11198.

Sugden MC, Holness MJ (2011) The pyruvate carboxylase-pyruvate dehydrogenase axis in islet pyruvate metabolism: going round in circles? Islets 3:302-319.

Taiwo YO, Bjerknes LK, Goetzl EJ, Levine JD (1989) Mediation of primary afferent peripheral hyperalgesia by the cAMP second messenger system. Neuroscience 32:577-580.

Tavares-Ferreira D, Ray PR, Sankaranarayanan I, Mejia GL, Wangzhou A, Shiers S, Uttarkar R, Megat S, Barragan-Iglesias P, Dussor G, Akopian AN, Price TJ (2020) Sex differences in nociceptor translatomes contribute to divergent prostaglandin signaling in male and female mice. Biol Psychiatry. Advance online publication. Retrieved February 15, 2021.

Usoskin D, Furlan A, Islam S, Abdo H, Lönnerberg P, Lou D, HjerlingLeffler J, Haeggström J, Kharchenko O, Kharchenko PV, Linnarsson S, Ernfors P (2015) Unbiased classification of sensory neuron types by large-scale single-cell RNA sequencing. Nat Neurosci 18:145-153.

Vasko MR, Habashy Malty R, Guo C, Duarte DB, Zhang Y, Nicol GD (2014) Nerve growth factor mediates a switch in intracellular signaling for PGE2-induced sensitization of sensory neurons from protein kinase A to Epac. PLoS One 9:e104529.

Walker JS, Carmody JJ (1998) Experimental pain in healthy human subjects: gender differences in nociception and in response to ibuprofen. Anesth Analg 86:1257-1262.

Wang C, Gu Y, Li G-W, Huang L-YM (2007) A critical role of the cAMP sensor Epac in switching protein kinase signalling in prostaglandin E2induced potentiation of P2X3 receptor currents in inflamed rats. J Physiol 584:191-203.

Wang S, Dai Y, Fukuoka T, Yamanaka H, Kobayashi K, Obata K, Cui X, Tominaga M, Noguchi K (2008) Phospholipase C and protein kinase A mediate bradykinin sensitization of TRPA1: a molecular mechanism of inflammatory pain. Brain 131:1241-1251.

Wing MR, Bourdon DM, Harden TK (2003) PLC-epsilon: a shared effector protein in Ras-, Rho-, and G alpha beta gamma-mediated signaling. Mol Interv 3:273-280.

Zwick M, Davis BM, Woodbury CJ, Burkett JN, Koerber HR, Simpson JF, Albers KM (2002) Glial cell line-derived neurotrophic factor is a survival factor for isolectin. J Neurosci 22:4057-4065. 